\title{
Oper oder Experiment
}

\author{
Walsers Romane im Musiktheater
}

Zwei Romane von Robert Walser haben bisher zu musikdramatischen Auseinandersetzungen angeregt: einerseits der letzte, 1925 entstandene und erst 1972 posthum erschienene »Räuber«-Roman (SW 12 bzw. AdB 3, 9-150), andererseits der Tagebuch-Roman Jakob von Gunten (SW 11). Ersterer inspirierte Johannes Harneit und Michel Roth zu experimentellen Arbeiten, in denen sie traditionelle Komponistenrollen befragen und sich an den Rändern dessen bewegen, was noch dem Theater zugeordnet werden kann. Jakob von Gunten wiederum spielte schon bei der ersten großen musikalischen Beschäftigung mit Walser, Wladimir Vogels Dramma-Oratorio Flucht, eine zentrale Rolle und diente später als Vorlage für musikdramatische Werke von Benjamin Schweitzer und Helmut Oehring.

\section{1 $\quad$ Einleitung}

Von den vier Romanen, die sich von Walser erhalten haben, ${ }^{1}$ wurden bisher nur zwei für das Musiktheater dramatisiert. Die Gründe, weshalb Geschwister Tanner und Der Gehülfe zwar in Film und Theater(vgl. Kap. 8.2-3), nicht aber für die Oper bearbeitet wurden, könnten inhaltlicher, aber auch opernpraktischer Art sein. Für eine Oper braucht es eine nachvollziehbare Handlung sowie wenige gut konturierte Hauptrollen mit klaren Stimmfächern und allenfalls einen Chor. Das vielstimmige Geschwisterdispositiv der Geschwister Tanner eignet sich wenig für ein Opernlibretto. ${ }^{2}$ Bei Der Gehülfe müsste umgekehrt eine komplexe Familiengeschichte gesungen werden, die allein schon wegen der Kinderrollen den Rahmen der Oper sprengt. ${ }^{3}$ Der Roman Jakob von Gunten kann einfacher in die Parameter der Oper eingebunden werden, während der

1 Der Tobold-Roman (verfasst 1918) und der Theodor-Roman (verfasst 1921) sind verschollen (vgl. RWH, 166-168 und 173-175).

2 Nur das winterliche Ende des Romans wurde musikalisch rezipiert, vgl. die Ausführungen zu »... hinaus in die Winternacht.« von Christophe Schiess (Kap. 11.21).

3 Insbesondere die an Silvi verübten Grausamkeiten könnten nicht ohne Zynismus auf einer Theaterbühne >gespielt $<$ werden. 
»Räuber«-Roman mit seiner extrem selbstreflexiven Erzählweise nach neuen Theaterformen ruft.

Sowohl zu Jakob von Gunten als auch zum »Räuber«-Roman ist literaturwissenschaftlich und -kritisch viel publiziert worden. Der enigmatische Charakter der Romane mit zahlreichen von Walser gezielt gesetzten Leerstellen fordert die Interpretierenden geradezu heraus, des labyrinthischen Textes mit unterschiedlichen Bezugssystemen habhaft zu werden. Zugänge zu den Texten werden aus ganz unterschiedlichen Perspektiven und Theoriehintergründen freigelegt.

\subsection{Zur Dramatisierung von Jakob von Gunten}

Anders als der alle traditionellen Erwartungen an einen Roman enttäuschende »Räuber«-Roman ist Jakob von Gunten trotz der zahlreichen Selbstreflexionen als Tagebuch linear angelegt, wodurch an viele Theatertraditionen angeknüpft werden kann. Deshalb eignet sich dieser Roman gut für Dramatisierungen auf dem Sprechtheater, ${ }^{4}$ im Théâtre musical (vgl. dazu die Umsetzung von Astride Schlaefli in Kap. 8.5.3) und der Oper. Allerdings erzwingt die Übertragung auf das Theater schmerzhafte Eingriffe in die eigentlichen Qualitäten des Romans: Dramatische Momente, die Walsers vielzüngiger Tagebuchschreiber elegant und schwerelos umschifft, müssen hervorgehoben werden. Reto Sorg, der Jakob von Gunten in den politischen Kontext der europäischen Vorkriegskultur stellt, beschreibt die entdramatisierenden, antitheatralischen und postdramatischen Verfahren treffend:

Wenn Jakob sein Lebensglück bedenkt, ist sein Lebensunglück bereits in vollem Gang. Der schleichende Niedergang des Instituts, der als Schwundform einer Katastrophe erscheint, bildet dazu den passenden Rahmen. Da der utopischapokalyptische Zeitraum aufgehoben und mit dem Diesseits der Erzählgegenwart überblendet wird, fehlt für das Szenario einer drohenden Katastrophe die Grundlage. Die fehlende Fallhöhe manifestiert sich auch darin, dass der metaphorische Überschwang [...] entfällt und sich das Pathos der Darstellung weniger auf die äußere Bewegung der Handlung als vielmehr auf die inneren Regungen Jakobs erstreckt. Indem das Institut Benjamenta eine Bildungsutopie verkörpert und diese zugleich ironisch negiert, gleicht es einer Heterotopie, wie sie Michel Foucault beschrieben hat, einem beunruhigenden >Gegenort<, durch den die Gemeinplätze einer Gesellschaft »gleichzeitig repräsentiert, bestritten

4 Die jüngste Dramatisierung auf dem Sprechtheater ist jene von Barbara Frey (Regie) und Amely Joana Haag (Dramaturgie). Schauspielhaus Zürich (Premiere am 20.05.2017). 
und gewendet sind, gewissermaßen Orte außerhalb aller Orte, wiewohl sie tatsächlich geortet werden können. $\aleph^{5}$

Sorgs Beobachtung macht deutlich, welche Probleme entstehen, wenn Jakob von Gunten für das Theater bearbeitet wird: Das Dialogische tritt in den Vordergrund, die Romanfiguren bekommen Körper, sprechen und singen, die handlungsbezogenen Elemente werden wichtig. Erstaunlicherweise erfüllt der Roman nach solch selektiver Behandlung viele Rahmenbedingungen einer traditionellen Theaterform wie der Oper. Es gibt einen zentralen Spielort: die Dienerschule von Herrn Benjamenta; es gibt einen überblickbaren Zeitraum mit einem klaren Anfang: Jakobs Eintritt in die Dienerschule; und es gibt einen klaren Schluss, da Jakob das Tagebuchschreiben beendet (»Aber weg jetzt mit der Feder. Weg jetzt mit dem Gedankenleben.«SW 11, 164) und ankündigt, mit Herrn Benjamenta in die Wüste ziehen zu wollen. Die Handlung des Romans ist vergleichsweise einfach und könnte als Zerfall einer >totalen Institution< im Sinne Erving Goffmans (vgl. Kap. 7) bezeichnet werden: ${ }^{6}$ Die anfänglich auf Zucht und Disziplin ausgerichtete Dienerschule, deren Unterricht hauptsächlich aus dem Auswendiglernen der Vorschriften besteht, löst sich zunehmend auf, bis sie ihren Betrieb einstellt.

Die Hauptfiguren von Jakob von Gunten erscheinen wie das verfremdete Dispositiv einer Opera seria mit fünf tragischen Figuren und einem lieto fine, einem letztlich glücklichen Schluss. Allerdings sind die Paarbildungen der Opera seria ins Verwandtschaftliche verschoben: Das >Königspaarく ist ein Geschwisterpaar, nämlich Herr Benjamenta und seine Schwester Lisa. Sie führen zusammen das Institut Benjamenta, in dem den Knaben eine professionelle Unterordnung beigebracht wird, die sie befähigt, später als Diener arbeiten zu können. Das >Konfliktpaar bilden Kraus und Jakob von Gunten, aber sie streiten nicht - wie meist in der Oper - um eine Frau oder einen Mann, sondern um unterschiedliche Vorstellungen von Disziplin und Unterwerfung: Während Kraus den Zielen des Instituts Benjamenta nachstrebt, geht Jakob zunehmend seinen eigenen Weg und beschleunigt damit die Auflösung der `totalen Institution< Benjamenta. Die außenstehende fünfte Figur ist nicht wie in der Opera seria eine verstoßene, unglücklich verliebte oder sich rächende Person, sondern Jakobs Bruder Johann, der außerhalb des Instituts und auch der anderen Hauptfiguren steht. Er hat es in die oberste Gesellschaftsschicht gebracht und demütigt Jakob noch viel radikaler (»Du

5 Sorg: Abschied von Europa?, S. 173; Verweis im Zitat auf Foucault: Andere Räume, S. 39.

6 Vgl. Goffman: Asyle, S. 15-23. 
bist jetzt sozusagen eine Null«; SW 11, 66), als dies Herr Benjamenta und die Mitschüler tun.

Sogar ein höchst verfremdetes lieto fine findet statt, aber nicht wegen einer Hochzeit oder eines Friedensschlusses, sondern weil Jakob dem Werben von Benjamenta nachgibt und mit dem Tagebuchschreiben aufhört. Erst sein Verstummen ermöglicht das ziemlich wackelige >Happy End des Romans. Für Reto Sorg ist dies eine Anspielung auf den Schluss von Georg Büchners Erzählung Lenz (»So lebte er hin«), Bernhard Malkmus erkennt darin die letzte Bestätigung der novela picaresca, weil Benjamenta und Jakob wie Don Quijote und Sancho Pansa in die Wüste ziehen. ${ }^{7}$

Wie in vielen Opern gibt es im Roman Jakob von Gunten auch so etwas wie einen Chor, nämlich jenen der sieben Zöglinge (Kraus und von Gunten nichteingerechnet), die zwar einzeln aufgeführt werden, aber in den Dramatisierungen als meist polyphoner Chor behandelt werden. Im Roman selber gibt es noch einen zweiten, quasi stummen Chor, nämlich die geheimnisvollen Lehrer, die sich in einem totenähnlichen Schlafzustand befinden. Diese Lehrer-Episode wird nur bei Benjamin Schweitzer berücksichtigt. ${ }^{8}$

\subsection{Benjamin Schweitzers Vorstudie zur Oper}

Der 1973 in Marburg geborene und später in Lübeck aufgewachsene Benjamin Schweitzer absolvierte sein Musikstudium - nur wenige Jahre nach dem Mauerfall - in Dresden. Dort traf er auf Lehrer, die nicht zur experimentellen Avantgarde zählen (Komposition bei Wilfried Krätzschmar, Musiktheorie bei Jörg Herchet und Dirigieren bei Christian Kluttig), dafür aber für ihr solides Handwerk und ihre stilistische Offenheit bekannt sind. Weitere Studien führten Schweitzer zu Paavo Heininen in Helsinki. Seither beschäftigt er sich mit Fennistik und ist neben dem Kompositionshandwerk als Übersetzer tätig.

Schon 1992, in seinem letzten Schuljahr, hatte er sich mit Jakob von Gunten beschäftigt. 1996 begann er noch während seiner Ausbildung mit der Komposition der Oper. Es handelt sich um Schweitzers erste Auseinandersetzung mit Musiktheater; ihr folgten später eine ganze Reihe weiterer Werke, wobei Schweitzer gerne verschiedene Genres erprobt, zum Beispiel den Tanz einbezieht und jüngst auch eine in Jamaika spielende Operette komponiert hat. $^{9}$

7 Vgl. Sorg: Abschied von Europa?, S. 174; RWH, 117.

8 Schweitzer:Jakob von Gunten, 2. Akt, Nr. 21, Szene VII, S. 152-165 (Partitur).

9 Südseetulpen (Libretto Constantin von Castenstein), UA 14.01.2017, Opernhaus Chemnitz. 
Parallel zu den vorbereitenden Arbeiten zur Oper Jakob von Gunten schrieb Benjamin Schweitzer den 20-minütigen Zyklus Und ging (1997) mit Texten von Walser und Ausschnitten aus Carl Seeligs Wanderungen mit Robert Walser. Das halbszenische Werk in zehn Teilen für Bariton, Sprecher, Violine, Horn und Schlagzeug kann als Vorstudie zur Oper verstanden werden, weil Schweitzer hier jene Elemente erprobt, die in der KammeroperJakob von Gunten konstitutiv sein werden: die Verbindung zwischen Sprechen und Singen sowie die karge Instrumentierung, die kaum begleitende oder unterstützende Funktion übernimmt, sondern meist wie ein Kommentar wirkt, wie eine zweite oder dritte Stimme zum gesprochenen oder gesungenen Wort.

In der Operngeschichte gibt es seit der Semiopera, der Opéra comique und dem Singspiel eine lange Tradition der Verbindung von Sprechen und Singen. Die gesprochenen Teile treiben die Handlung voran, die gesungenen Teile bilden in Form von Arien, Liedern, Ensembles und Chören musikalische Tableaus, die den dramatischen Verlauf rhythmisieren. Mit Vorläufern im 19. Jahrhundert, beispielsweise den Melodramen von Robert Schumann, wird im 20. Jahrhundert das Verhältnis zwischen Sprechen und Singen neu befragt und in vielen Kompositionen thematisiert - am pointiertesten und ins Theologische gesteigert in der Oper Moses und Aron von Arnold Schönberg: Moses, der Stotterer, der die Wahrheit Gottes unbeholfen verkündet, darf bei Schönberg nur sprechen; sein älterer Bruder Aron, der diese den Menschen mit Bildern vermittelt und sie zum Tanz ums goldene Kalb verführt, ist bei Schönberg der Sänger. Dieses Paradox, dass Schönberg dem Gesang in Moses und Aron nur die Verführung zutraut, nicht aber die eigentliche prophetische Wahrheit, ist zu einem zentralen Topos in der Kompositionsgeschichte des 20. Jahrhunderts geworden, der sich auch in zahlreichen Walser-Vertonungen niederschlägt.

Für beide Werke von Schweitzer ist diese Ambivalenz von Singen und Sprechen zentral. Was in der Oper Jakob von Gunten ein ständiges Nebeneinander von Sprechen und Singen sein wird, ist im Zyklus Und ging streng geteilt. Relativ umfangreiche Ausschnitte aus Carl Seeligs Wanderungen werden von einem an einem Tisch sitzenden Sprecher vorgetragen; auch das vielvertonte frühe Gedicht Und ging (SW 13, 27) wird zum Schluss gesprochen, der Sänger »verläßt währenddessen ruhig und langsam den Raum «. ${ }^{10}$ Nur drei Teile des Zyklus' werden vom Bariton gesungen. Verbindend zwischen den beiden Welten von Sprechen und Singen fungieren drei Instrumente, nämlich Violine, Horn und Schlagzeug, wobei dem Schlagzeug die wichtigste Rolle zukommt. Dabei verzichtet Schweitzer auf alle Platteninstrumente, 
die Melodien bilden können (zum Beispiel Marimbas), und wählt stattdessen stark perkussive Instrumente wie Tempelblocks, Becken, Guero oder Bambusrasseln. Sie stehen für die konsonantische Welt des Sprechens. Violine und Horn hingegen korrespondieren mit der vokalen Welt des Singens, wobei Schweitzer auch bei den Melodieinstrumenten zahlreiche perkussive Strukturen verlangt. Nur gerade beim siebten Teil, der Vertonung des Gedichts Müdigkeit (SW 13, 28), bei dem Schweitzer das Schlagzeug weglässt, entsteht eine lyrische Grundstimmung.

Schweitzer arbeitet mit wenigen Mitteln, die er präzis und subtil einsetzt. Dies zeigt sich schon ganz am Anfang des Zyklus' bei der Vertonung des Gedichts Zeit (SW 13, 31f.), in dem das Wort »Zeit« das einzige Reimwort darstellt (vgl. Abb. 36). Schweitzer hebt die beiden ersten Verse des Gedichts mit tonalen Wechseln voneinander ab. Die Melodie des ersten Verses mit dem »Ich liege hier« entfaltet sich über dem as der Violine und die Töne passen sich weitgehend in einen B-tonartlichen Bereich ein: Nur das $h$ auf »hier« und das $d$ auf »Zeit« fallen aus dem Rahmen dieser diatonischen Melodie heraus. Der zweite Vers referiert auf das »ich sinne hier«: Das stützende as der Violine setzt aus und die Melodie liegt im Kreuzbereich. Zwar sind die ersten drei Töne nur um einen Halbton verschoben und auch der Gesamtgestus bleibt sich ähnlich, selbst der Zielton $d$ auf »Zeit« ist der gleiche. Und doch ist der Sinn der Melodie ein anderer, ein systemischer, denn die Melodie sinkt vom ais auf »hier« in Ganztönen zum $d$ auf »Zeit« ab: Das Spielerische, etwa die überraschenden Tritonus-Sprünge des ersten Verses $(f-h$ bzw. $a s-d)$, ist verschwunden; dafür gibt es einen konsequenten Abstieg, der auf $d$ schließt und mit den Ganztönen die anfängliche Diatonik beendet. Damit ist $d$ als Angelpunkt der Vertonung definiert; neun der zehn Wiederholungen des Wortes »Zeit« erklingen auf diesem Ton. Einzig das allerletzte Vorkommen >enttäuschtく sinnigerweise diese Konstruktion und endet auf $e$.

Die 1997 entstandene Komposition zeigt eine gründliche Beschäftigung mit Robert Walser: Späte und frühe Texte werden eingebunden, die Herisauer Zeit nimmt eine wichtige Rolle ein, zentrale Daten in Seeligs Wanderungen werden aufgenommen - so der Todestag an Weihnachten 1956, aber auch der 16. Mai 1943, an dem Robert Walser gegenüber Carl Seelig den Wunsch äußert, nur noch am Sonntag besucht zu werden. ${ }^{11}$ 


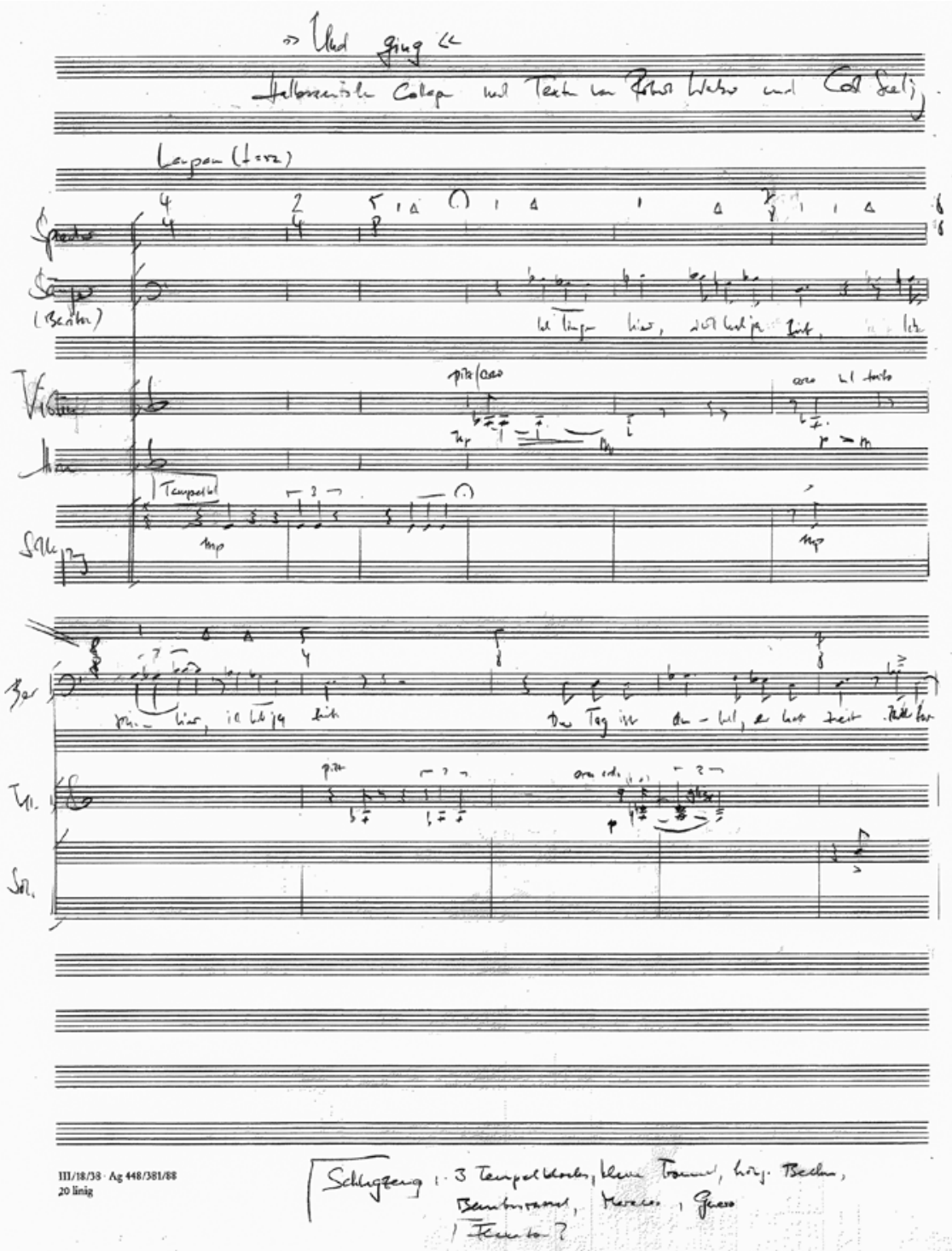

Abb. $36 \quad$ Benjamin Schweitzer: Und ging, S. 1 


\subsection{Walser-Mosaik}

Benjamin Schweitzers Oper Jakob von Gunten ist ein höchst komplexes Formgebilde, durch das ein Sprecher führt. Das Werk besteht aus drei Akten und 44 Unterabschnitten, wovon 19 als »Szene« hervorgehoben werden. Dazu kommen 19 instrumentale Zwischenspiele, insgesamt also 63 teilweise sehr kurze Elemente, die unterschiedlich komponiert und instrumentiert sind. Genau besehen sind es 62 Teile, weil das letzte Zwischenspiel und die letzte Nummer identisch sind: Die Differenzierung von Nummern und Zwischenspielen wird im offenen Schluss aufgehoben. Das eigentliche Romanende, das mit dem Abbruch des Tagebuchs zusammenfällt, lässt Benjamin Schweitzer in seinem Libretto weg. Auch die angedeutete Reise in die Wüste wird nicht erwähnt. Das Libretto endet mit Jakobs Bemerkung: »Doch erlauben Sie mir, Ihnen zu sagen, dass ich mich entschlossen habe, mit Ihnen zu gehen, wohin Sie wollen.« Danach sieht man, wie Jakob und Benjamenta packen und aufräumen, alles »sehr leise, mit ruhigen Bewegungen «. ${ }^{12}$ Schweitzer ersetzt Walsers offenen Schluss durch musikalische Aleatorik: Die bis dahin streng metrisierte Musik zerfällt; die drei noch nicht verstummten Instrumentalisten (Horn, Violoncello und Harfe) spielen in Feldern notierte Motive »in freier Anzahl und Reihenfolge und sollten dabei unregelmäßig zwischen den Feldern wechseln«. Die Gesamtlänge des Abschnitts kann je nach Regie zwischen mindestens 70 Sekunden und 5 Minuten dauern. ${ }^{13}$

Um eine überblickbare Form zu schaffen, gliedert Benjamin Schweitzer die komplexe Mosaikstruktur seiner Komposition in drei Akte. Der erste hat klassische Expositionsfunktion, der zweite weist einen leicht buffonesken Charakter auf und wirkt mit dem Auftritt von Jakobs Bruder Johann, der Rückblende zu den früheren Lehrern, vor allem aber mit dem Konflikt zwischen Jakob und Kraus wie ein Zwischenakt. Der letzte Akt gestaltet sich als zunehmender Zerfall bis hin zum Tod von Lisa und der immer offener und unverblümter geäußerten Liebesbezeugung von Herrn Benjamenta gegenüber Jakob. Parallel dazu verabschiedet sich ein Instrument nach dem anderen.

Der Sprecher nimmt in der Oper verschiedene Funktionen wahr. Besonders fallen die Bicinien auf, die Schweitzer zwischen dem Sprecher und Jakob gestaltet, zum Beispiel bei der Beschreibung der Großstadt (vgl. Abb. 37): Der Sprecher evoziert mit dem fünffachen Stabreim von »Geschiebe«, »Gedränge«, »Geschrei«, »Gestampf«, »Gesurr« das Getriebensein, in dem sich das Individuum nicht frei entfalten kann, während die Gesangsstimme

12 Schweitzer:Jakob von Gunten, S. 408f. Vgl. auch SW 11, 164.

13 Schweitzer:Jakob von Gunten, S. 411. 
in einer langen Kantilene die »fortlaufende Bewegung« ausdrückt und das Geschiebe und Gedränge auf einer Metaebene beschreibt. ${ }^{14}$ Dabei ist es die Sprechstimme, die die rhythmische Energie schafft und die Stelle periodisiert, zuerst mit zwei dreisilbigen, dann mit drei zweisilbigen Wörtern. Mit der melodielosen Sprechstimme können Walsers raffinierte, musikalischen Prinzipien folgende Stabreime und die in der Zweitsilbe folgenden vokalen Permutationen adäquat umgesetzt werden. Schweitzer setzt die Mittel und Wirkungen der Sprech- und der Singstimme im Sinne von Walsers Sprachkunst äußerst differenziert ein.

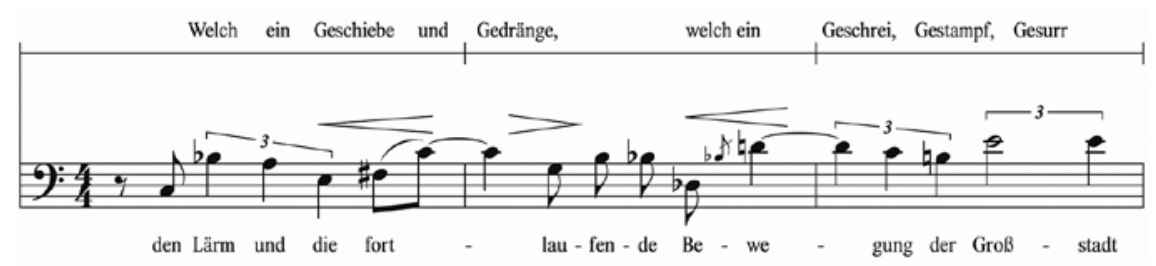

Abb. 37 Benjamin Schweitzer:Jakob von Gunten, 1. Akt, Nr. 15 (3 Takte nach Ziffer 59), Sprecher und Jakob (Partiturausschnitt)

Schweitzer tastet den Walser'schen Text seismografisch ab und setzt dann Wort und Musik wie Steine eines Mosaiks zusammen. Das führt zu einer Opernform, die entfernt an die frühe venezianische Oper erinnert, die sich auf reine Deklamation beschränkt, weder Arien noch längere Instrumentalstücke enthält und deshalb formal eher kleingliedrig konzipiert ist. Wie in der venezianischen Oper setzt auch Schweitzer die Szenen konstrastreich voneinander ab.

Dieses Formprinzip wird gleich am Anfang der ouvertürenlosen Oper deutlich (vgl. Abb. 38): Auf drei Partiturseiten finden zwei Nummern, ein Zwischenspiel und der Anfang von Nummer 3 Platz. ${ }^{15}$ Nummer 1 wird bloß mit Tempelblock spärlich begleitet und erinnert ganz direkt an Schweitzers Zyklus Und ging. Das Zwischenspiel exponiert auf der Marimba geradezu plakativ, nämlich in eine Oktave zusammengezogen, eine Zwölftonreihe. Sie ist in einem rätselhaften 7/8-Takt organisiert, der gehörsmäßig kaum nachzuvollziehen ist, weil der fürs Takt-Verständnis wichtige erste Taktschlag nicht ein einziges Mal erklingt. Schweitzer verlangt zudem in eckigen Klammern die interne Aufteilung des 7/8-Taktes in 4 + 3, aber auch hier wird der diese Aufteilung bestätigende fünfte Schlag kein einziges Mal hörbar. Man ist verführt,

\footnotetext{
14 Ebd., S. 78.

15 Ebd., S. 1-3.
} 

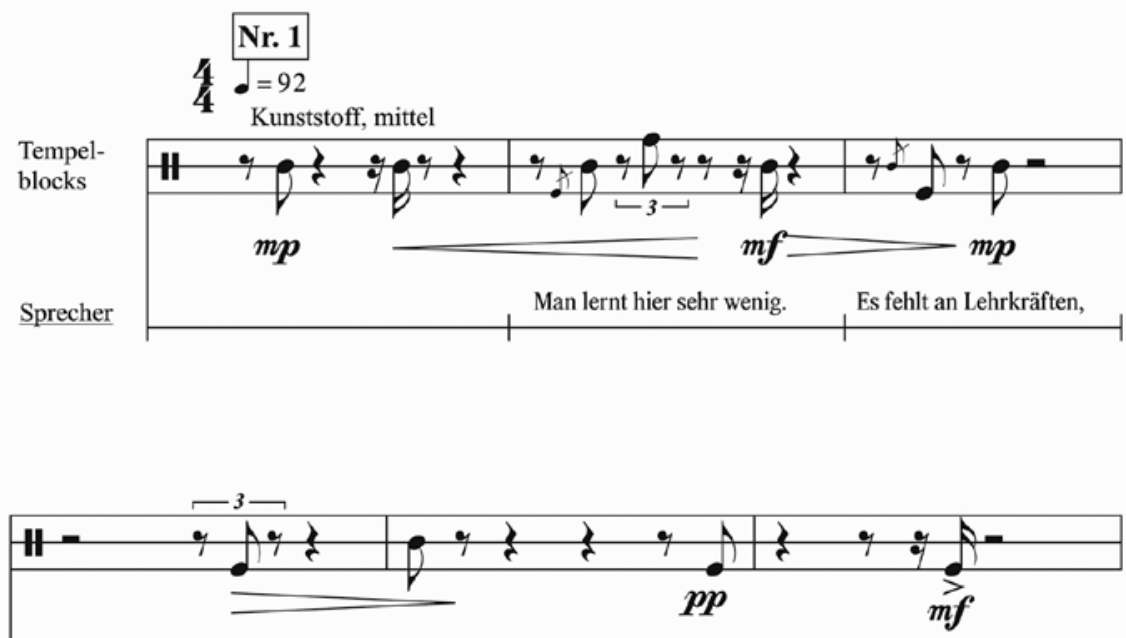

und wir Knaben vom Institut Benjamenta werden es zu nichts bringen, das heißt, wir werden alle
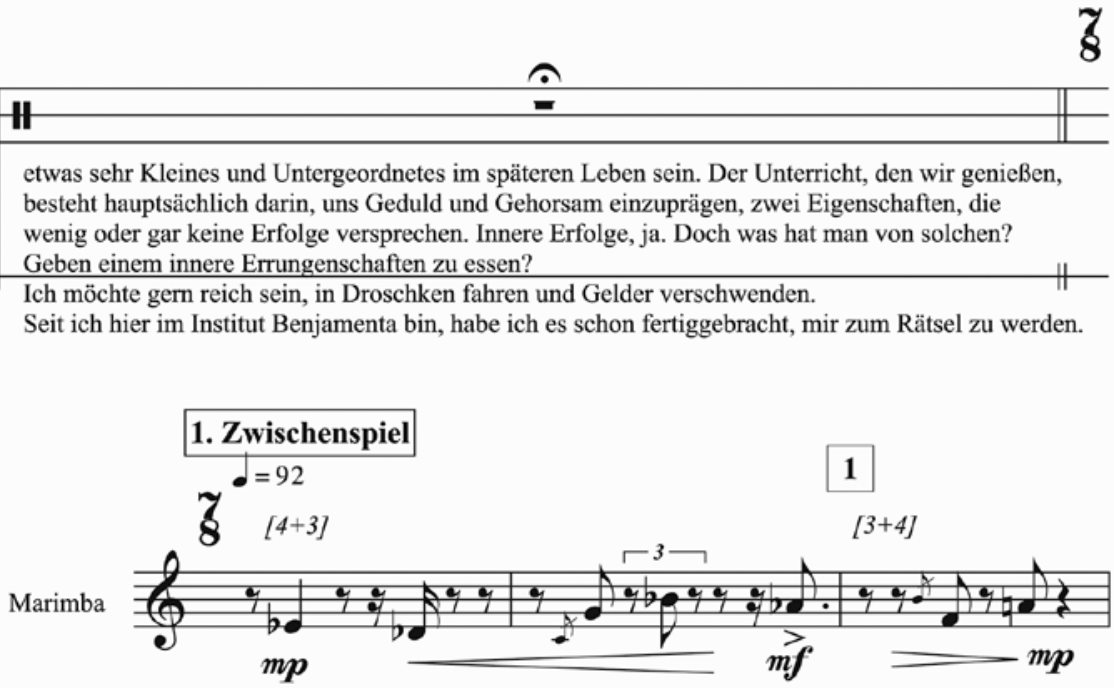

Abb. $3^{8}$ Benjamin Schweitzer:Jakob von Gunten, 1. Akt, Nr. 1-3 (Beginn) 

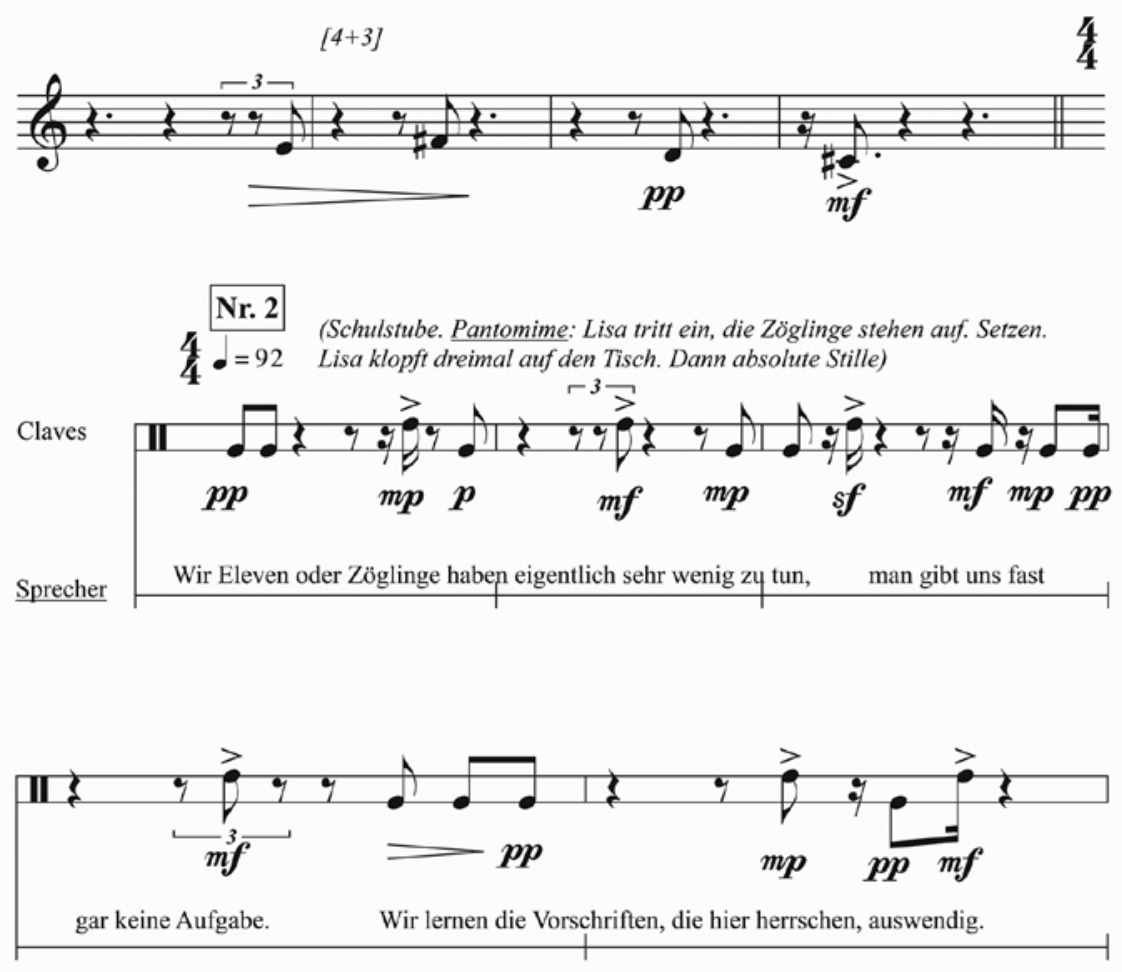

II

Oder wir lesen in dem Buch "Was bezweckt Benjamenta's Knabenschule?" Es gibt nur eine einzige Stunde, und die wiederholt sich immer: "Wie hat sich der Knabe zu benehmen?" Kenntnisse werden uns keine beigebracht. Es fehlt eben, wie ich schon sagte, an Lehrkräften, das heißt, die Herren Erzieher und Lehrer schlafen, oder sie sind tot, oder nur scheintot, oder sie sind versteinert, gleichviel, jedenfalls hat man gar nichts von ihnen. An Stelle der Lehrer unterrichtet und beherrscht uns eine junge Dame, die Schwester des Herrn Institutsvorstehers, Fräulein Lisa Benjamenta. Welch ein Unterricht! Doch ich würde lügen, wenn ich ihn kurios fände. Nein, ich finde das, was sie uns lehrt, beherzigenswert. Es ist wenig, und wir wiederholen immer, aber vielleicht steckt ein Geheimnis hinter all diesen Nichtigkeiten und Lächerlichkeiten. Irre ich mich? Weiß Gott, manchmal will mir mein ganzer hiesiger Aufenthalt wie ein unverstăndlicher Traum vorkommen. 


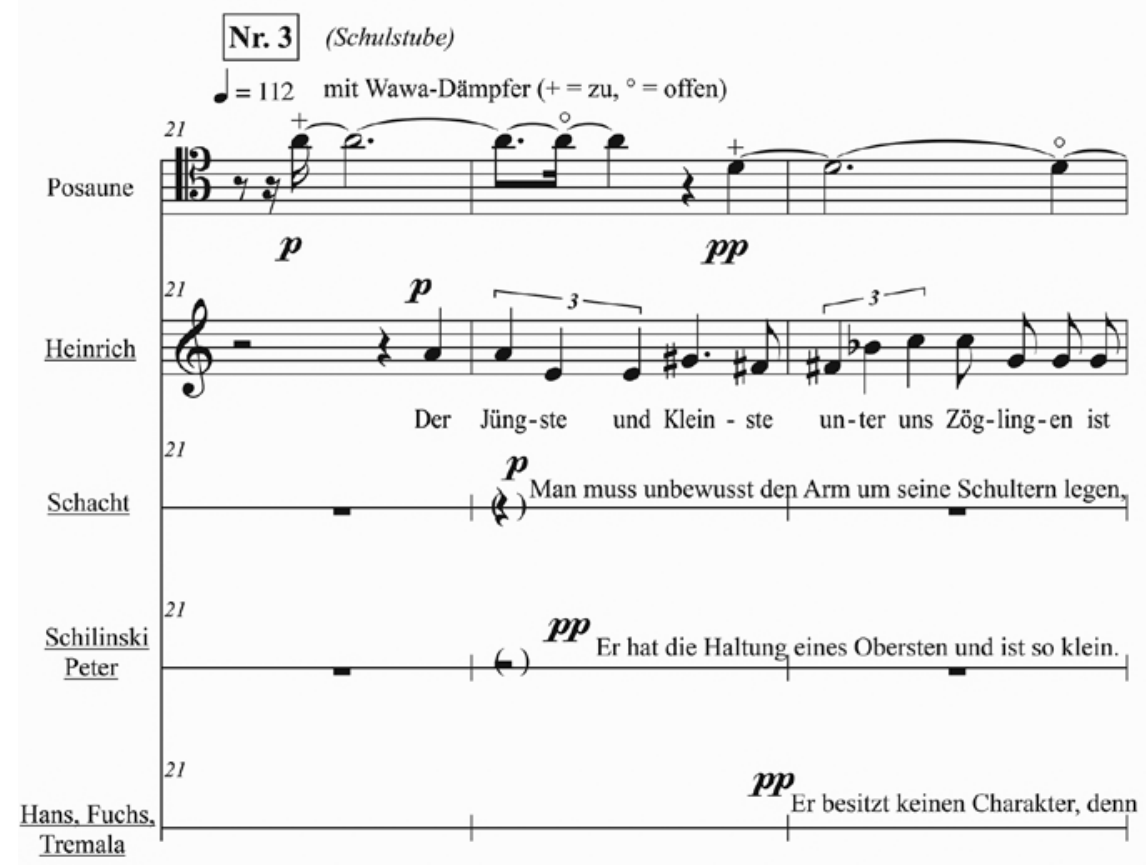

dieses Zwischenspiel als Gedankenmusik, als musikalischen Rebus aufzufassen: dreizehn Töne - das $c i s^{1}$ erklingt enharmonisch als $\operatorname{des}^{1}$ zweimal - für die dreizehn Rollen der Oper und der 7/8-Takt für die sieben Zöglinge. Die Einsatzabstände der ersten sieben Töne betragen sieben oder vier Sechzehntel, wobei sich eine Periodizität von zweimal $7-7-4$ Sechzehntel ergibt, einzig durch die Triole bei $b^{1}$ wird diese Regelmäßigkeit leicht verschoben.

Diese Reihe wird in einem auffälligen Moment der Oper, der Duo-Szene zwischen Lisa und Jakob, exponiert wiederholt, ebenfalls eingeleitet von der Marimba (vgl. Abb. 39). ${ }^{16}$ Allerdings wird hier die Reihe nicht in einer Oktave zusammengezogen, sondern die gesamte Tessitura des Jakob-Sängers verlangt, mehr als zwei Oktaven von $E$ bis $f i s^{1}$ in der Kopfstimme. Die Verteilung der zwölf Reihentöne ist kalkuliert auf die Bedeutung des dreizehn Silben umfassenden Textes bezogen; sieben Töne erklingen zu den fünf Silben von »Ich möchte reich sein « - für »reich « werden drei Reihentöne sausgegeben < mit den verbleibenden fünf Tönen wird »und den Kopf zerschmettert haben«

16 Ebd., S. 216. 
(acht Silben) gestaltet. Der riesige und im Piano zu realisierende Sprung vom $E$ zum fis $^{1}$ verschiebt die chiastische Aussage ins Widersinnige. Das $E$ liegt klar außerhalb des Umfangs eines hohen Baritons und kann nur im sogenannten Strohbassregister tonlos > geknarrt « werden, umgekehrt wäre das fis ${ }^{1}$ für einen hohen Bariton, der ein paar Töne zuvor ein $f^{1} \mathrm{im}$ Fortissimo gebracht hat, leicht mit Normalstimme erreichbar, aber Schweitzer will hier für die zentralen Worte »Kopf zerschmettert « den Wechsel in die kindlich-irr wirkende Kopfstimme.
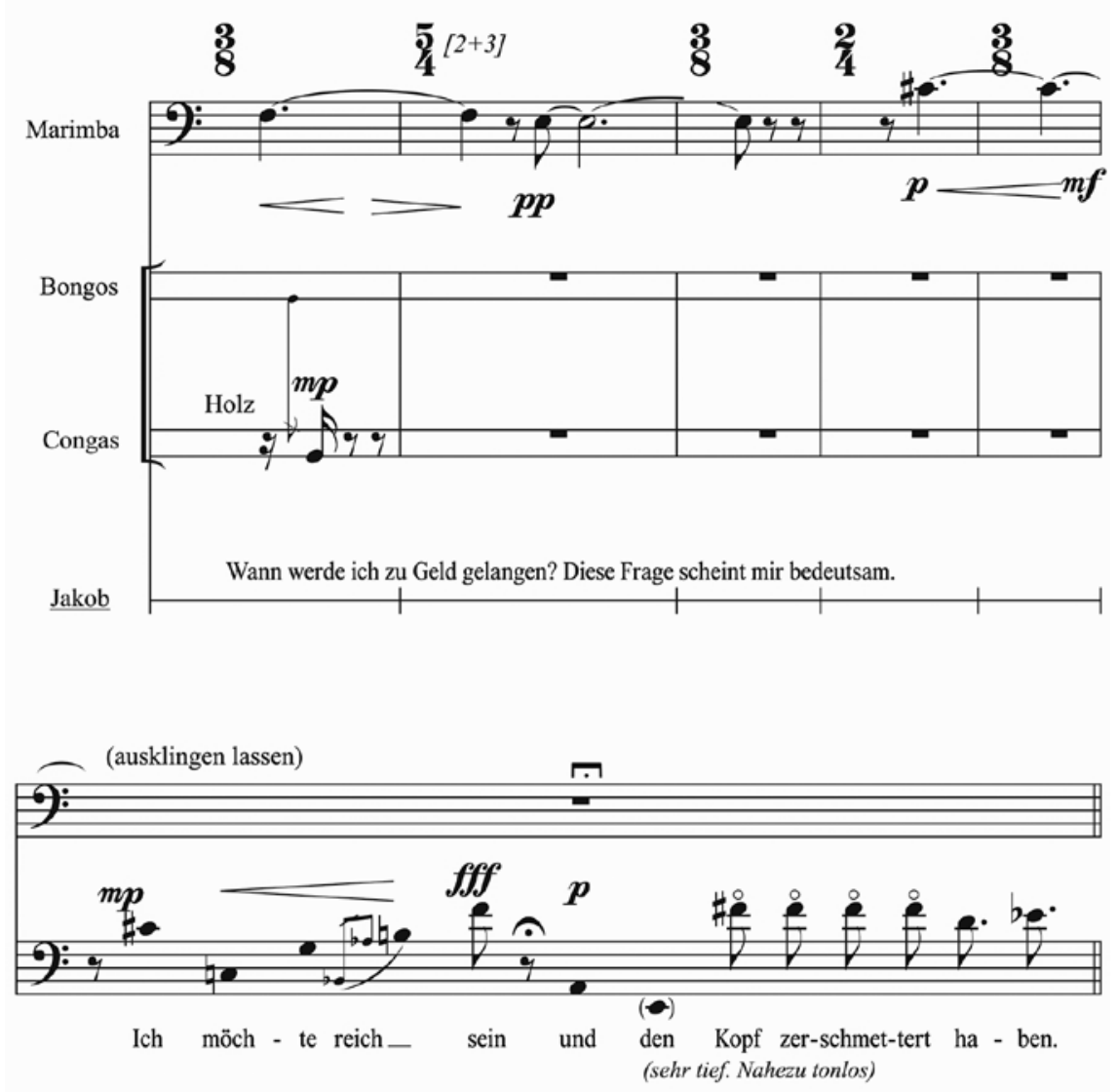

Abb. 39 Benjamin Schweitzer:Jakob von Gunten, 2. Akt, Nr. 25 - Szene X (1 Takt nach Ziffer 64) 


\subsection{Ungezwungene Reihen-Technik}

Andreas Sparberg hat anhand der Kompositionsskizzen von Benjamin Schweitzer die komplexe Tonhöhengrammatik der Oper aufgeschlüsselt. ${ }^{17}$ Dabei sind Zwölftonreihen wie die eben erwähnten eher ein Sonderfall. Daneben verwendet Schweitzer Zentraltöne, Intervallzyklen und Intervallreihen. Der Zentralton der Oper ist das cis für Jakob, der in nahezu allen Szenen präsent ist. Benjamentas Zentralton ist das $g$; der Tritonus zum cis markiert das dissonante Oppositionsverhältnis zwischen Jakob und Benjamenta. Lisas Zentraltöne sind $e$ und $b$, die beide im Kleinterzverhältnis sowohl zu Jakob als auch zu Benjamenta stehen. Lisas Kleinterz symbolisiert Trauer und Rückzug. Die Zentraltöne von Kraus sind fis und gis; sie umrahmen mit Quarten Jakobs cis und charakterisieren die Aggressivität und stramme Unterwerfung von Kraus.

In der gesamten Tonhöhenorganisation wählt Schweitzer eine offene Diastematie, die Intervalle können - wie die Zentraltöne von Lisa und Kraus nach oben oder unten geführt werden. Der Tritonus als nicht umkehrbares Intervall stellt ein starres Verhältnis dar.

Neben den Zentraltönen sind den einzelnen Figuren Intervalle zugeordnet. Jakob ist mit der kleinen Sekunde (Spannung) charakterisiert, der Chor der Zöglinge mit einer Großterz (dem Chorintervall), Lisa und Kraus auch hier mit der Kleinterz bzw. der Quarte und Benjamenta erneut mit dem nicht umkehrbaren Tritonus. Die Auswahl der Intervalle ist von Schweitzer zusätzlich differenziert; so verfügt Jakob über den gesamten Intervallvorrat, allen anderen Figuren >fehlen< Intervalle. Der Tritonus kommt exklusiv bei Jakob und Benjamenta vor. Der Chor der Zöglinge nutzt bloß zwei Intervalle, die große Sekunde und die große Terz. Genau diese beiden >gewöhnlichen< Intervalle, die sonst bei allen Personen vorkommen, werden bei der sünften< Figur dieser 〉Opera seria<, dem aufstrebenden Bruder Johann - der für Schweitzer »zur heimlichen Hauptperson des Stückes $\aleph^{18}$ wird - ausgespart. Johann wird damit schon im Intervallvorrat als snobistischer Außenseiter charakterisiert.

Andreas Sparberg legt in seiner Arbeit dar, wie Schweitzer aus diesen Intervallen, die den Figuren zugeordnet sind, Intervallreihen ableitet, bei denen zwar die Intervallfolge, nicht aber deren Diastematie festgelegt ist. Diese Intervallreihen wiederum werden auf vier verschiedene Weisen permutiert, indem je nachdem jeder zweite oder jeder dritte Ton weggelassen werden kann.

17 Sparberg: Benjamin Schweitzers Oper Jakob von Gunten, S. 48-6o.

18 Ebd., S. 51. 
All diese Verfahren veranschaulichen, wie sehr Schweitzer gegen die klassischen Gesetze der Dodekaphonie verstößt, wo die Zwölftonreihe die gesamte Tonhöhenstruktur dominiert. Zentraltöne, freie Diastematie und Permutationen mittels Weglassen von Tönen sind ihr fremd. Schweitzer gewinnt mit den verschiedenen Kompositionstechniken letztlich eine enorme Freiheit, die es ihm erlaubt, in jedem Moment auf Walsers Sprache reagieren zu können. Und weil es paradoxerweise doch auch >echte Zwölftonreihen für die Hauptfiguren gibt, steht ihm an speziellen dramatischen Höhepunkten auch dieses kompositorische Mittel noch zur Verfügung. Man mag sich fragen, weshalb Schweitzer es sich so kompliziert macht, indem er determinierende Vorordnungen entwickelt, um diese dann so lange zu permutieren, bis sie ihren Charakter als Vorgabe verlieren und der Komponist letztlich jenseits aller Systeme kompositorische Entscheide fällen kann. Dieses Verfahren korrespondiert allerdings mit dem Walser'schen Erzähler, der sich am Tagebuch festhält, in alle Richtungen abschweift, sich immer wieder zur Selbstdisziplin mahnt und doch alle Freiheiten herausnimmt - inszenierte Zwänge, um sie zwanglos umgehen zu können.

Andreas Sparberg zeigt auch das auffällige Instrumentationssystem auf, bei dem die Instrumente wie obligate Hauptstimmen in der Barockoper sowohl den Sprecher als auch die singenden Protagonisten begleiten. ${ }^{19}$ Schweitzer gliedert die 18 Spielpositionen in drei Ensembles und ordnet sie den Figuren genau zu. Es gibt ein Ensemble für die Hauptfiguren, ein Ensemble für die Zwischenspiele, in dem die Hauptfiguren Stellvertreter-Instrumente bekommen, und schließlich ein Ensemble für die Zöglinge:

$\begin{array}{lll} & \text { Hauptensemble } & \text { Zwischenspiel-Ensemble } \\ \text { Jakob: } & \text { Violoncello } & \text { Bratsche } \\ \text { Sprecher: } & \text { Geräusch-Schlagzeug } & \text { Klangschlagzeug } \\ \text { Lisa: } & \text { Klarinette } & \text { Flöte } \\ \text { Benjamenta: } & \text { Horn } & \text { Harfe } \\ \text { Kraus: } & \text { Fagott } & \text { Bassklarinette } \\ \text { Johann: } & \text { Tenorsaxophon } & \text { Tenorsaxophon }\end{array}$

Ensemble der Zöglinge: 1. und 2. Violine, Kontrabass, Oboe, Trompete, Posaune, Pauke

Das Hauptensemble spiegelt die Dominanz der Männerstimmen im Solistenensemble mit der Klarinette als einzigem Diskant-Instrument. Die Stellvertreter-Instrumente in den Zwischenspielen weisen durchwegs einen >weicheren< Klang auf als die Hauptinstrumente, und das Ensemble als

19 Ebd., S. 36-40. 
Ganzes wird von Sparberg als »ausgesprochen impressionistisch ausgerichtet « bezeichnet. ${ }^{20}$ Das Ensemble der Zöglinge nimmt sich äußerst heterogen aus, enthält Instrumente aus allen Instrumentengruppen, die sich zudem mit Ausnahme der beiden Violinen äußerst schlecht mischen.

Auch dieses Verfahren der semantisch-dramaturgischen Differenzierung jedes Instruments erinnert an die frühe venezianische Oper, bei der es noch kein eigentliches Orchester gab und die Instrumente zur koloristischen Unterstützung der Protagonisten oder der szenischen Topoi verwendet wurden. Mit dem Wissen um diese Differenzierung wird auch Schweitzers Schluss nochmals verstärkt. So wie im Roman gegen Ende der Zerfall des Instituts fortschreitet, so verstummen bei Schweitzer die Instrumente nach und nach, bis nur noch das Cello (Jakob) und die Herrn Benjamenta wie zwei gegensätzliche Seelen definierenden Instrumente Horn und Harfe übrigbleiben. Das Horn steht für Jagd, Macht und Natur, die Harfe für Traum und die Sehnsucht nach einer anderen Welt. Für Schweitzer ist Herr Benjamenta jene Figur, die »wirklich in sich zerrissen ist «; $;{ }^{21}$ deshalb charakterisiert er ihn mit diesen zwei sehr unterschiedlichen Instrumenten. Dieses merkwürdige Trio ist gewissermaßen Schweitzers neue >Wüste $\triangleleft$, welche an die Stelle von Walsers Wüste tritt, die im Stück weggefallen ist.

\subsection{Schwierige Rezeption}

Diese Einblicke in die Partitur lassen vermuten, wie anspruchsvoll es ist, die karge und höchst komplexe Oper von Benjamin Schweitzer auf die Bühne zu bringen. Die Uraufführung am 9. Oktober 2000 im Theater der neben Dresden liegenden Stadt Meißen wurde von der Hochschule für Musik Carl Maria von Weber, Dresden, realisiert und fand im Rahmen der 14. Dresdner Tage der zeitgenössischen Musik statt. Studierende der Instrumental- und Opernklasse spielten und sangen; Titus Engel, der Schweizer Dirigent und damals wie Benjamin Schweitzer Student in der Dresdner Dirigierklasse von Christian Kluttig, gab mit Jakob von Gunten sein Operndebut; es war der Start zu seiner internationalen Dirigentenkarriere. Der langjährige Leiter der Dresdner Opernklasse Andreas Baumann führte Regie.

\footnotetext{
$20 \quad$ Ebd., S. 39. Das Tenorsaxophon erscheint in beiden Ensembles und dient als klangliches Bindeglied.

21 Zitiert nach ebd., S. 40.
} 
Zweimal wurde die Oper seither in professionellen Theatern gezeigt, nicht zufälligerweise in den Schweizer >Walser-Städten< Biel ${ }^{22}$ und Sankt Gallen ${ }^{23}$ (neben Herisau gelegen). In diesen beiden Folgeproduktionen waren die Regieteams bemüht, den Stoff zu beleben und aus dem 95 Minuten langen Mosaik >richtiges< Theater zu machen. Die Sache sollte >rübergebracht< werden, wie das im Theaterjargon heißt. Rainer Holzapfel inszenierte in Biel die Oper als absurde Groteske, bei der mit theaterwirksamen Bildern auf Direktheit gesetzt und die bei Walser und Schweitzer nur angedeutete Erotik voll ausgespielt wurde. Mit Ausnahme von Jakob und Lisa waren fast alle Figuren krass überzeichnet. In Sankt Gallen dekonstruierte der damals vor allem als Filmregisseur tätige Johannes Schmid die Oper im Sinne des postdramatischen Theaters und verwandelte sie in ein visuelles Spektakel, das in einem Zelt vor dem Theater gegeben wurde.

Beide Ansätze fanden bei der Kritik wenig Anklang. Zur Bieler Aufführung schreibt Jürg Huber:

Denn bald wird die zu Beginn angelegte Vielschichtigkeit aufgegeben zugunsten dramatischer Stringenz, und Walsers assoziativ schillernde Vorlage wird verengt zu einem linear erzählten Drama, das an handfester Deutlichkeit nichts zu wünschen übrig lässt. [...] Wo Walser andeutet, herrscht nun Gewissheit, wo Walser in der Schwebelässt, [entlädt sich das] latente Aggressionspotenzial unter den Zöglingen [...] immer wieder in gewalttätigen Aktionen. Doch Walsers Text widersetzt sich der dramatischen Vereinfachung. So zeigt der wenig plausible Schluss der Oper - wie ist es zu erklären, dass sich Jakob entschliesst, beim eindimensional als lüsterner Päderast gezeichneten Institutsleiter zu bleiben? - die Schwäche dieser Adaption auf. ${ }^{24}$

Noch schärfer kritisiert Michelle Ziegler die Sankt Galler Produktion:

Gänzlich grobschlächtig kommt Johannes Schmids Inszenierung daher. In ihrem Versuch, auf das Ausdeuten zu verzichten, beschränkt sie die visuellen Mittel auf eine Drehbühne mit Kanalisationsschächten, aus denen die Zöglinge emporsteigen. Warum Schmid dennoch plumpe Effekte wie am Zeltdach kreisende Lichter einbringt, ist unverständlich. ${ }^{25}$

22 Theater Biel/Solothurn, Premiere in Biel am 14.09.2002, Premiere in Solothurn am 21.09.2002. Regie: Rainer Holzapfel; musikalische Leitung: Franco Trinca; Bühnenbild und Kostüme: Franziska Kaiser.

23 Premiere Theater St. Gallen am 15.04.2010. Regie:Johannes Schmid; musikalische Leitung: Christian Schumann; Bühnenbild und Kostüme: Michael S. Kraus.

24 Huber: Hermetische Enge, S. 64.

25 Ziegler: Entzauberter Romanheld, S. 53. 
Beide Regie-Ansätze >übertönten ` die bewusste Kargheit der Partitur und ihre kammermusikalische Differenziertheit. Jürg Huber zur Bieler Produktion:

Auch die Musik vermag keine weitere Dimension hinzuzufügen. Die spröde Klanglichkeit verdichtet sich nur in der grossen Szene der Lisa Benjamenta (Edith Weiger), als diese Jakob vergeblich zu verführen sucht und sich dabei ins eigene Fleisch schneidet. Sonst halten die Klänge aus dem Orchestergraben Distanz zum Bühnengeschehen. Der musikalische Zusammenhang mag auf dem Papier gegeben sein; ohrenfällig wird er nicht. ${ }^{26}$

Auch Michelle Ziegler erwähnt in ihrer Kritik zur Sankt Galler Produktion, wie sehr die Szene mit Lisa - »[d]er einzige volle Klang des Abends « - als dramatischer Höhepunkt heraussticht, und beschreibt die Musik sonst als einen »Klangteppich aus kleinen Partikeln, der sich [...] seltsam distanziert und scheinbar unabhängig von der Handlung entwickelt. «27

Wer trägt die Schuld an dieser schwierigen und kritischen Rezeption? Geht Benjamin Schweitzers Konzept, auf eine vereinnahmende Vertonung im Sinne einer traditionellen Oper zu verzichten und damit möglichst nahe an Walsers Text zu bleiben, einfach nicht auf? Müsste ihm eine sattere Orchestration empfohlen werden, also nicht nur ein einziges orchestrales Fast-Tutti bei Lisas Liebeserklärung, das beide Kritiken positiv erwähnen?28 Darf man die Singstimmen so ungestützt vom Orchester agieren lassen? Oder liegt es an der Regie, die taub ist für die Zwischentöne der Oper? Oder ist es schließlich doch ein weiteres Mal Robert Walser selber, der sich letztlich jedem Konzept oder Zugriff verweigert, wie es Martin Walser so treffend feststellt: »Robert Walser schlägt einem von Mal zu Mal die Instrumente kaputt, mit denen man ihn erklären will.«29

Obwohl der Komponist mit allen drei Inszenierungen zufrieden war und die unterschiedlichen Ansätze schätzte, ${ }^{30}$ müsste Schweitzers Oper vielleicht doch in einer experimentellen Theaterform gezeigt werden, die gar nicht mehr nach >richtigem< Theater strebt und das Szenische nicht als Primärreiz versteht. So könnte man das Orchester, das bei Schweitzer das Bühnengeschehen

26 Huber: Hermetische Enge, S. 64.

27 Ziegler: Entzauberter Romanheld, S. 53.

28 Bei diesem Fast-Tutti (nur Tenorsaxophon und Violine II fehlen) handelt es sich um die 12. Szene bzw. Nummer 32 (3. Akt, Ziffer 2-24). Sie wird von einem Solo der Marimba eingeleitet (nach Ziffer 2), das tonhöhenmäßig und in der Oktavlage identisch ist mit dem ersten Zwischenspiel der Oper (vgl. Abb. 38).

29 Walser: Alleinstehender Dichter, S. 148.

30 E-Mail von Benjamin Schweitzer an Roman Brotbeck vom 21.02.2021. 
nicht - wie traditionellerweise in der Oper - stützt, sondern es vielmehr spiegelt, auf die Bühne stellen als Teil des Bühnenbilds, in dem die Darsteller agieren; vielleicht könnten die Instrumentalisten sogar zu sich bewegenden Akteuren werden. Mit einem derartigen Vorgehen würden die Kargheit der Partitur und die engen Beziehungen zwischen Instrumenten und Sängern nicht überspielt, sondern in ihrer Sinnhaftigkeit sichtbar gemacht.

\subsection{Helmut Oehrings Gebärdenmusik}

Zehn Jahre nach Schweitzer setzte sich Helmut Oehring im Werk Gunten (2008) im Auftrag des Gare du Nord Basel und des Ensemble Phoenix Basel mit dem Roman Jakob von Gunten auseinander. Oehring wurde 1961 als Sohn gehörloser Eltern kurz vor dem Mauerbau in Ostberlin geboren; er begann erst mit vier Jahren zu sprechen. Mit 25 Jahren - in diesem Alter beendete Benjamin Schweitzer seine Walser-Oper - lernte Helmut Oehring Noten lesen und sie auf der Gitarre nachspielen. Voraus ging eine wilde, schwierige, aber auch sehr reiche Jugendzeit in der DDR, aus der Oehring als Komponist bis heute vieles schöpfen kann. Der Musik näherte sich Oehring in der DDR über das, was von der Rockmusik damals zu hören war, und später über Konzerte zeitgenössischer Musik. Zu seinem 5o. Geburtstag verfasste er eine Autobiografie, in der er mit durchaus literarischen Qualitäten und Verfahrensweisen sein Leben und dessen Niederschlag in seinen Werken schildert. Mauerfall und Wende spielen darin keine wesentliche Rolle, denn von der DDR hatte er sich innerlich schon vorher als Schulversager, jugendlicher Pyromane, Heckenschütze mit Luftgewehr und Wehrdienstverweigerer konfliktreich abgewandt. In gewisser Weise bildete das Jahr 1990 für Oehring den Höhepunkt seiner DDR-Karriere und zugleich das Ende einer fast drei Jahrzehnte dauernden autodidaktischen Suche nach einer musikalischen Sprache, als er - ohne jede formale musikalische Ausbildung - von Georg Katzer als Meisterschüler an der Berliner Akademie der Künste aufgenommen wurde. Georg Katzer war neben Friedrich Goldmann, der Oehring ebenfalls förderte, einer der einflussreichsten Komponisten und Lehrer der DDR. Er wurde auch im Westen gespielt und verfügte über ein internationales Netzwerk in Ost und West.

Ohne Georg Katzer und seine klar aber freundlich formulierte Bitte auf einer Postkarte, endlich einmal anzufangen mit der Notenarbeit, würde ich heute noch mumifiziert vor Furcht und dieser Leere in der Birne vor einem weißen leeren Notenblatt sitzen. Als Underdog und Nononononame komponiert es sich leichter, weil kaum jemand sich für dich interessiert. Seit dem Moment, in dem 
ich mitgeteilt bekam, dass ich als Meisterschüler der Akademie der Künste zu Berlin in die Klasse von Georg Katzer aufgenommen war, hatte ich Schnappatmung vor Stolz und Ladehemmung. ${ }^{31}$

Schon als Kind wird er zum Übersetzer zwischen der gehörlosen Welt seiner Eltern und jener der Hörenden. Zur Stimme seiner Eltern schreibt er: »Stimme Papa. Wie kaputte Bremsbeläge. Oder mittelgroße Kreissäge. Langgezogene Töne in der oberen Lage eines Altsaxophons. Stimme Mama. Bassklarinette. Tiefe Lage. Um H herum. «32 Für Oehring ist die Gebärde bis heute die Muttersprache. Und er denkt und fühlt seine Musik in Gebärden. Darauf führt Oehring auch seine Vorliebe für das experimentelle Musiktheater zurück, bei dem sich Gebärden und Klang verbinden. Die Erfahrung eines Treffens gehörloser Kinder und Jugendlicher im Saalbau Friedrichshain, wo extra eine Band engagiert wurde, nur weil die Hörenden das auch so tun, ist für Oehring im Rückblick das Schlüsselerlebnis für sein musiktheatralisches Schaffen: »Vermutlich wurden genau hier die ersten Weichen gestellt auf den musiktheatralischen Schienen, auf denen ich damals wie heute ohne ordentlich abgestempelten Fahrausweis fahre. «33

Jazz, Rock und zeitgenössische Musik sind in Oehrings Entwicklung gleichwertige Einflussbereiche, aber auch Sport und insbesondere das Boxen faszinieren ihn. Diese Einflüsse sind in Oehrings vielfältigem CEuvre überall präsent. Der Einbezug Gehörloser, vor allem der ebenfalls in der DDR geborenen und aufgewachsenen Gebärdensolistin Christine Schönfeld, prägt bis heute zahlreiche von Oehrings Kompositionen. Immer wieder will er den Klangraum der gehörlosen Welt, zu der die Stille genauso wie der >Lärm < gehört, in seinem oft multimedialen Musiktheater kompositorisch verarbeiten. Schon in der Jugend spielt die Lautstärke eine große Rolle:

Ich werde nie vergessen können, wie ich an meinem Schlagzeug saß, bestehend aus nur einem Becken, aber super montiert auf einem Ständer. Ich öffnete die Fenster. Alle sollten mich hören und wissen, was ich kann. Ich drosch darauf ein, bis die Schlägel zersplitterten, das Becken verbeult war und die Nachbarn Sturm klingelten. Meine Mutter saß zwei Meter entfernt seelenruhig neben mir und strickte blaue Pullover mit Zopfmuster in der Brustmitte. Silbriges Klippklapp war zu hören, wenn mein Becken schwieg. Alles LalaLand. ${ }^{34}$

31 Oehring: Mit anderen Augen, S. 55 .

32 Ebd., S. 17.

33 Ebd., S. 22.

34 Ebd., S. 38. 
Das Beispiel zeigt, dass die Stille nur den Gehörlosen selbst gehört: »Der Geräuschpegel im Alltag der Gehörlosen ist brutal. Es hört ja niemand, wie die Tasse auf den Tisch scheppert oder die Tür knallt.« ${ }^{35}$ Gerade Oehrings frühe Werke zeichnen sich durch extreme Lautstärken und krude Titel (zum Beispiel Do you wanna blow job für Saxophonquartett) aus, so als müsste die Stille in der Kommunikation mit seinen Eltern und gehörlosen Freunden überschrien werden.

Nach der langen Inkubationszeit ist Oehring nach 1990 zu einem äußerst produktiven Komponisten geworden. Sein CEuvre - oft in interdisziplinären Produktionskollektiven entstanden - umfasst über 400 Werken, viele davon abendfüllend. Allerdings: Eine Oper in traditionellem Sinne gibt es von ihm keine.

\subsection{Das explizite Theater von Helmut Oehring}

In Helmut Oehrings Gunten wird nichts versteckt oder bloß angedeutet, sondern alles möglichst explizit gemacht: Die Präsenz aller Instrumentalisten auf der Bühne ist ausdrücklich verlangt; die Musikerinnen und Musiker spielen - gekleidet in Internatsanzüge des frühen 20. Jahrhunderts - die Zöglinge aus Walsers Roman. Oehring komponierte das Werk ursprünglich für das Ensemble Phoenix Basel und schrieb einzelnen seiner Mitglieder die Partie förmlich auf den Leib, zum Beispiel dem exzentrischen Kontrabassisten Aleksander Gabryś (vgl. Kap. 11.16) oder dem Pianisten und musikalischen Leiter des Ensembles Jürg Henneberger. Auch bei dieser Produktion entstand der Eindruck eines kreativen Missverständnisses. So schreibt Sigfried Schibli in der Basler Zeitung zu Gunten lakonisch: »Starke Stoffe erzeugen nicht immer starkes Musiktheater. Helmut Oehring scheitert in der Gare du Nord mit seinem >Gunten< - aber mit Anstand.« Gerade die Ausrichtung auf das Theatralische hat Schibli missfallen: »Markus Bothes Regie macht aus dem von Jakob verehrten >Fräulein< eine nervende Zicke (authentisch: Christine Urspruch) und aus dem Schulleiter Herrn Benjamenta (Georg Martin Bode) einen schmierigen Alt-Schönling «. ${ }^{36}$

Helmut Oehring ist in der musikalischen Walser-Rezeption ein Sonderfall, weil er weder vor noch während der Kompositionsarbeit die Biografie oder andere Werke von Robert Walser kannte. In der DDR wurde Walser wenig rezipiert. Die Schulmeinung war, dass Walser »die bürgerliche Ideologie zwar

35 Ebd.

36 Schibli: Der Abkömmling, S. 7 . 
kritisch analysiere, dieser aber letztlich verhaftet bleibe, da er keine Lösung zu deren Überwindung entwerfe« (RWH, 415). Rein zufällig - so die Aussage des Komponisten bei einem Werkgespräch ${ }^{37}$ - sei er auf Walsers Roman gestoßen und sei vom Text und seinen zeitgenössischen Bezügen sofort fasziniert gewesen. Im gleichen Gespräch äußerte Oehring auch, wie sehr ihn die Bekanntheit von Robert Walser und dessen hohe Wertschätzung in der Schweiz überrascht habe. Dieses Nicht-Wissen erklärt die überraschende und für Walser-Kenner teilweise befremdende musikalische Kontextualisierung in Gunten. Oehring zitiert nämlich die großen Sinfoniker Richard Strauss und Gustav Mahler und damit eine mondäne und hochkulturelle Welt, die Walser fremd war. Insbesondere der von Oehring eingeführte Mahler-Bezug erweist sich aber als verblüffend evident: Der Schluss von Gunten wird musikalisch dominiert von einer a-cappella-Version des 1901 komponierten MahlerLiedes »Ich bin der Welt abhanden gekommen «, 38 die Jürg Henneberger für das Ensemble Phoenix arrangiert hat. ${ }^{39}$ Das Lied spielt in Mahlers eigenem Schaffen eine zentrale Rolle und ist in der Rezeption gleichsam zum Emblem für Mahlers Leben und Komponieren geworden. ${ }^{40}$ Eine zusätzliche welthistorische Dimension erhielt das Lied, als dessen Manuskript im Jahr 2000 bei Sotheby's Wien versteigert werden sollte und aufflog, dass es sich dabei um Raubgut handelte, welches sich der in der Nachkriegszeit dominierende österreichische Musikwissenschaftler Erich Schenk aus dem Nachlass des Musikwissenschaftlers Guido Adler widerrechtlich angeeignet hatte. ${ }^{41}$

Ich bin der Welt abhanden gekommen,

Mit der ich sonst viele Zeit verdorben,

Sie hat so lange von mir nichts vernommen,

Sie mag wohl glauben, ich sei gestorben. ${ }^{42}$

37 Podium von Roman Brotbeck mit dem Komponisten anlässlich des Berner Gastspiels beim Musikfestival Bern, am 18. September 2009 in der Dampfzentrale Bern.

38 Mahler: Lieder nach Texten von Friedrich Rückert.

39 Bereits 1983 hatte der Komponist und Chorleiter Clytus Gottwald das Mahler-Lied für 16 Stimmen a cappella bearbeitet.

40 Vgl. Kinderman: $» I c h$ bin der Welt abhanden gekommen«; Hefling: The compositions of »Ich bin der Welt abhanden gekommen«; Partsch: »Ich bin der Welt abhanden gekommen«.

41 Schenk arbeitete während des >Dritten Reiches` eng mit Herbert Gerigk zusammen und schrieb auch Artikel für dessen Lexikon der Juden in der Musik. Vgl. Sakabe: Erich Schenk und der Fall Adler-Bibliothek; dies.: Die Bibliothek von Guido Adler. Vgl. auch das HörFeature von Lissek/Stratka: ich bin der welt abhanden gekommen. 
Helmut Oehring erkannte im Lied von Gustav Mahler nicht nur die inhaltliche Ähnlichkeit zu Walser - Friedrich Rückerts erste Gedicht-Zeile kann als emphatisches Synonym des lakonischen »beiseit« $(S W$ 13, 22) von Walser verstanden werden - sondern auch die direkte Verbindung von Walsers Sprache zum stilistisch changierenden Ton Gustav Mahlers, bei dem Innigkeit bis zum Kitsch und dessen ironische Brechung zusammenfallen. Theodor W. Adornos Diktum zu Mahler in seiner Wiener Gedenkrede von 1960 könnte denn auch auf Walser übertragen werden: »Seine musikalische Sprache selber ist durch und durch gebrochen. Sie fordert jenes Convenu von der Musik als einer Kunst reiner Unmittelbarkeit heraus. «43 Die Mahler-Stelle in Gunten ist ein einmaliger Moment, weil zwei Welten, die scheinbar nichts miteinander zu tun haben, plötzlich wie Puzzlestücke ineinanderpassen.

In seiner Autobiografie beschreibt Oehring, wie er sich schon während der DDR-Zeit ohne Rücksicht auf Verluste auf Neues stürzte und sich von Unbekanntem und Unerwartetem mitreißen ließ, dieses in seinem Sinne interpretierte und sich zu eigen machte. ${ }^{44}$ Dieses Vorgehen ist auch bei seiner Beschäftigung mit Walsers Jakob von Gunten zu beobachten. Das Andeutende, Verweisende, das Fantasierte des Romans wird in Oehrings Theateradaption weggewischt und der Realistik etwa von Frank Wedekinds Frühlings Erwachen (1891) angenähert. Die Beziehungen von Jakob, Lisa und Benjamenta sind als Ménage-à-trois dargestellt. Wie schon bei Wladimir Vogels Bearbeitung des Romans musste für eine solche Interpretation Lisa Benjamenta am stärksten umgedeutet werden. Allerdings wird diese nicht wie bei Vogel zu Jakobs Anima im Sinne von Jungs analytischer Psychologie veredelt, sondern zur Nymphomanin gewandelt, die schon in der zweiten Szene zu Jakob ins Bett steigt, in der vierten Szene einen Orgasmus erlebt und in der siebten den gehorsamen Kraus mit dem zur Peitsche umfunktionierten Mikrofon zum Cunnilingus zwingen will.

Die drastischen Erweiterungen, welche die Vertreter des Regietheaters zur Musik von Benjamin Schweitzer imaginierten, schreibt Oehring also gleich in die Partitur hinein, so als müssten die oft verhüllten erotischen Andeutungen des Romans demaskiert und unverblümt vorgeführt werden, worum es im Roman >eigentlich $<$ geht.

43 Adorno: Mahler, S. 325.

44 Vgl. Oehring: Mit anderen Augen, S. 43-58. Dort beschreibt der Komponist die Werkgenese von Coma I für Orchester (1991) als Reflexion des Staats->Komas< der DDR, des eigenen Gitarren-Duos Koma (1988), der Komas bei Boxkämpfen und des Komas des »damals weltbesten Handballers Joachim Jo Deckarm« (S. 55) nach einem schweren Sportunfall am 23. März 1979. 
Auch beim von Stefanie Wörnemann entwickelten Libretto wird kein komplexer Formaufbau gesucht, sondern eine einfache Revue gezeigt. In der Mitte der Bühne steht ein Standmikrofon, in das Jakob regelmäßig wie ein Leadsänger hineinspricht. Die Titel der Szenen erinnern an ein Jugendbuch, bei dem die Abenteuer des Protagonisten kapitelweise erzählt werden. Und wer Oehrings autobiografische Ausführungen zu seiner traumatischen Schulund Lehrzeit kennt, kann sich des Eindrucks nicht erwehren, dass er in seinen Gunten auch viel eigene Lebenserfahrung eingebracht hat.

Das Musiktheater besteht aus elf Szenen und drei Traumhörspielen:

\author{
Szene 1 Jakob kommt \\ Szene 2 Jakob träumt \\ Traumhörspiel 1 Jakob sieht Lisa (Mysterium-Traum) \\ Szene 3 Jakob macht Männchen \\ Szene 4 Jakob wildert \\ Szene 5 Jakob versteht nicht ganz \\ Traumhörspiel 2 Jakob marschiert (Soldaten-Traum) \\ Szene 6 Jakob schweigt und schweigt \\ Szene 7 Jakob in Ägypten \\ Szene 8 Jakob kotzt \\ Szene 9 Jakob tanzt \\ Szene 10 Jakob lacht \\ Szene 11 Jakob lebt weiter \\ Traumhörspiel 3 Jakob geht (Entscheidungs-Traum)
}

Die drei >Traumhörspiele sind offene Zonen, in denen das Explizite des Theaters verschwindet. Das letzte Hörspiel bildet den Schluss des Stücks, der ähnlich offenbleibt wie das aleatorische Ende bei Benjamin Schweitzer. Das zweite Hörspiel schließt an die Caféhaus-Szene an, in der Jakob von seinem Bruder Johann gedemütigt wird. Diese Szene wird von lauter werdenden und zunehmend deformiert klingenden Caféhaus-Geräuschen begleitet, sodass Johann und Jakob sich schließlich anschreien müssen. Die im >SoldatenTraum < evozierte Soldatenmusik wird vom Ensemble übernommen und bildet einen der wenigen längeren musikalischen Schwerpunkte des Stücks.

Das erste Hörspiel setzt nach der Begegnung von Jakob und Lisa ein und leitet zum ersten musikalischen Zitat über: Die Zöglinge stimmen alle in unterschiedlichen Sprachen den Kanon Bruder Jakob an. Auf einer ersten Ebene ist das eine vom Namen Jakob abgeleitete >Walser'sche< Assoziation, aber vermutlich klingt auch schon hier Gustav Mahler an, der im dritten Satz seiner ersten Sinfonie mit diesem Kanon einen grotesk-ironischen Trauermarsch gestaltet, der mit Kontrabass in hoher Lage, Fagott, Violoncello und Basstuba einsetzt. Der Kanon Bruder Jakob korrespondiert ebenfalls mit 
dem erneut von Jürg Henneberger ausgewählten und für a-cappella-Chor gesetzten Guggisberglied. In der Partitur verlangt Oehring bloß »(Schweizer) Volkslied (mit Jodeln) « und in der Szenenanweisung vermerkt er: »Jakob geht während des Liedes wie Schlagerstar zum vorderen Bühnenrand, singt zum Publikum, befestigt am Ende Handmikro wieder am Stativ $\ll_{0}{ }^{45}$ Diesem etwas >touristisch anmutenden Wunsch Oehrings nach einem Zitat von Schweizer Klischees wirkte Henneberger mit der Wahl des abgründigen und tieftraurigen Guggisberglied entgegen. Es gelang ihm - auch wegen des >Laien<-Chors aus nicht ausgebildeten Stimmen der Mitglieder des Ensemble Phoenix - jenen einfachen und doch innigen Ton zu finden, den Walser in eigenen Texten zur Musik so sublim beschreibt. ${ }^{46}$ Das Lied gilt als das älteste Schweizer Volkslied ${ }^{47}$ und schafft am Ende der dritten Szene einen ähnlichen Ruhepunkt wie später das Mahler-Lied.

\subsection{Multimedia contra Monodrama}

Oehrings Musik ist geprägt von ostinater Motorik, die zuweilen fast obsessiv homophon komponiert ist. Dabei sucht er nach einer komplexen Synchronizität der Instrumentalstimmen, die ihm erlaubt, mit den wenigen Instrumenten eine satte orchestrale Wirkung zu erzielen. Oehring komponiert auch einige Solos und vor allem viele Duos. Das steht in engem Bezug zur szenischen Situation. In der sechsten Szene - Jakob schweigt und schweigt - wird zum Beispiel die Episode mit Schachts Geschlechtskrankheit behandelt. Schacht, der Viola-Spieler, legt sich zu Jakob ins Bett und dieser möchte sich das erkrankte Organ genauer ansehen, was Schacht ablehnt. Diese intime Situation wird von einem langen Duo von Viola und Violoncello begleitet. Es ist technisch ausgesprochen schwierig zu spielen, nicht nur wegen der rhythmischen Koordination und weil der Bratscher wegen seiner szenischen Aktionen auswendig spielen muss, sondern weil Oehring die beiden Instrumente fast durchwegs in Oktaven spielen lässt. Er wählt also jenes Intervall, bei dem man sofort die kleinste intonatorische Trübung hört: eine musikalische Selbstähnlichkeit als Symbol für die enge Verbindung von Schacht und Jakob, die das vertrauliche Wissen um die venerische Erkrankung zusammenschweißt. Selbstverständlich ist es zudem eine hochvirtuose, im gestisch-diastematischen wie dynamischen Verlauf äußerst theaterwirksame Musik. Synchronizität hat

45 Oehring: Gunten, Partitur, S. 19.

46 Zum Beispiel in Die Kapelle (SW 4, 98f.) oder Die Handharfe (SW 4, 139).

47 Von Greyerz: Das alte Guggisberg Lied. 
immer etwas Virtuoses; wenn zwei oder drei das Gleiche tun, fällt das im Alltag und auf der Bühne sofort auf. Dabei ist Oehring kein Tüftler, der an einem musikalischen Detail lange herumschleift; seine Gunten-Partitur ist rasch komponiert, gewisse Ausarbeitungen überließ er dem Dirigenten und andere Stellen klärten sich erst in der Probenphase. Auch hat er keine Skrupel, Außermusikalisches, zum Beispiel den erwähnten Caféhaus-Lärm, einzubeziehen.

Es ist insbesondere dieser unbekümmerte Umgang mit den Mitteln des konventionellen Theaterspiels, der Schiblis Diktum des >mit Anstand gescheiterten Komponisten begründete. Mit der Wahl von Christine Urspruch als Lisa Benjamenta wurde diese theatralische Ebene noch zusätzlich verstärkt, weil die Schauspielerin sehr stark mit ihrer Rolle als Alberich im Münsteraner Tatort identifiziert wird. Dieser trivialkulturelle Effekt wurde nicht für eine doppelbödige Interpretation von Lisa Benjamenta genutzt, sondern als reiner Effekt ausgespielt. Wie Urspruch waren auch die anderen Schauspieler und Schauspielerinnen in ihren Rollen fixiert und in diesem Korsett zu keiner dramatischen Entwicklung fähig.

Sechs Jahre nach der Uraufführung wurde Oehrings Gunten neu vom Wiener Ensemble PHACE inszeniert. ${ }^{48}$ Mit einem Kniff umging die Neuinszenierung die Ambivalenzen der Uraufführung: Der Regisseur Steffen Jäger verzichtete weitgehend auf das Theaterspiel. Ein einziger Schauspieler, Tim Breyvogel, sprach und spielte kongenial die drei Hauptrollen Jakob, Benjamenta und Lisa. Auf einem kleinen Podium, kaum größer als ein Dirigentenpodest, wirkte er wie eingeschlossen. Die Musiker trugen keine Kostüme, sondern spielten in schwarzer Konzertkleidung. Größere Partien, etwa jene des Bruders Johann oder jene von Kraus, wurden auf mehrere Ensemble-Mitglieder verteilt, die den Text teilweise in ihrer Muttersprache vortrugen. Das von Oehring angestrebte Chaos der Mittel wurde radikal reduziert und in ein japanisch anmutendes Ritualtheater gewandelt. Dieser vollständige Verzicht auf szenische Effekte erzeugte eine enorme dramatische Spannung: im Zentrum der wahnwitzige Monolog des Schauspielers mit sich selbst, das Tagebuchschreiben umgestülpt zu einem expressionistischen Entäußerungsakt, das Ensemble ein fast scheuer und betroffener Begleiter, als würde es einem Menschenversuch beiwohnen. In dieser Szenerie konnte sich der Groove von Oehrings motorischer Musik in seiner ungeschlachten Kraft frei entfalten. Diese Zweitproduktion zeigt, wie Musiktheater - wie so oft in der Oper - durch Verzicht auf >Theater $<$ neu interpretiert werden kann.

48 Wiener Konzerthaus, Berio-Saal, 25./26. September 2015, Regie: Steffen Jäger, Bühnenbild: Sabine Freude. PHACE Series 15/16 - $\mathrm{N}^{\circ}{ }_{1}$. 


\subsection{Zwei gegensätzliche Opern und ihre Berührungspunkte}

Gegensätzlichere Komponisten als Benjamin Schweitzer und Helmut Oehring sind schwer vorstellbar. Deshalb überraschen die zahlreichen Parallelen und Überschneidungen zwischen den beiden abendfüllenden Walser-Werken. Das Quintett der Hauptfiguren steht in beiden Kompositionen im Zentrum. Schweitzer bleibt dabei im Rahmen der Oper: alle Hauptfiguren sprechen und singen; Oehring entscheidet sich konsequent für das gesprochene Musiktheater und differenziert die Rollen weiter: Für das zentrale Dreieck von Lisa Benjamenta, Jakob von Gunten und Herrn Benjamenta verlangt er professionelle Schauspieler, Kraus wird vom Kontrabassisten gespielt, Bruder Johann vom Pianisten. Auch die Zöglinge sind in beiden Werken als Chor dargestellt, der mit eigenen Szenen bedacht ist. Beide Komponisten verzichten auf einen reinen Männerchor und führen Frauenstimmen bzw. Hosenrollen ein. Bei Benjamin Schweitzer besteht der Chor aus einem professionellen Gesangsensemble, er wählt hier also die Mittel der Oper, während Oehrings ZöglingsChor in der Tradition des Théâtre musical von den Instrumentalistinnen und Instrumentalisten performt wird. Auch die zunehmend erotisch aufgeladene Handlung wird in beiden Kompositionen dargestellt, allerdings in unterschiedlichen Modi: Für sexuelle Szenen wählt Schweitzer das Mittel der Pantomime (zum Beispiel für den Besuch bei einer Prostituierten oder den Griff an Jakobs Geschlechtsteil); Oehring umgekehrt macht auch die implizite Sexualität bei Walser durchwegs explizit. Beide Werke sind kammermusikalisch geprägt. Benjamin Schweitzer schreibt zwar für ein Kammerorchester mit 18 Positionen, verwendet aber meist nur wenige Instrumente. Ein richtiges Tutti gibt es in der ganzen Oper keines, einzig die Szenen mit der Liebeserklärung und dem Tod Lisas sind größer besetzt und verdichten sich zu dramatischen Höhepunkten. Oehrings Besetzung beschränkt sich auf acht Instrumente; trotzdem wirkt sein Stück über weite Strecken orchestraler als Schweitzers Oper.

\subsection{Schwierige Dramatisierung des »Räuber«-Romans}

Im Unterschied zuJakobvon Gunten, bei dem es Dialoge, Personen unterschiedlichsten Charakters, Haupt- und Nebenfiguren und eine nacherzählbare Handlung gibt, erscheint der »Räuber«-Roman gegen jede Form von Dramatisierung gewappnet. Zwar könnte man verführt sein, die Liebessehnsüchte des Räubers nach Wanda bzw. Edith - die auch jene des Erzählers sind - szenisch zu einer boulevardesken Dreieckskomödie umzufunktionieren, die - statt mit C. F. Meyers berühmtem Schuss von der Kanzel - mit Ediths Schuss auf die 
Kanzel sogar eine dramatische Pointe aufwiese. Aber man würde damit an Walser vorbei eine sentimentale Geschichte konstruieren, deren Possenhaftigkeit der Roman gerade thematisiert:

»Hier sank er um. Ein leiser Schrei durchschnitt die hohe Halle. Edith stand hochaufgerichtet. Ihren Händen entglitt ein Revolver. Die Kanzeltreppe hinab tröpfelte kostbares Räuberblut. Nie wurde intelligenteres Blut vergossen. >O hochgradig Intellektueller und zugleich Dummers, flüsterte Wanda. Einige Herren umringten achtungsvoll die stumme Rächerin.« $\left(\mathrm{AdB}_{3}, 143\right)$

Für Peter Utz verhindert diese Szene »[u]nter Aufbietung aller trivialer Mittel [...] den Absturz des Räubers in ein trivial gewordenes Identitätsmodell« und bewahrt »den $>$ Räuber -Roman davor, als Bildungsroman zu enden. $\aleph^{49}$

Nicht einmal zu einem Zweipersonenstück - der Erzähler und sein Räuber könnte man den Roman umarbeiten. Die beiden Protagonisten Erzähler und Räuber sind derart ineinander verwachsen, dass es eines einzigen Schauspielers mit zwei Gesichtern oder zwei Mündern bedürfte. Aber auch dies trifft nicht zu, weil es die Autorschaft zementiert, die der Roman gerade auflöst.

Sehr schön hat W. G. Sebald in seinem Walser-Essay die radikal autofiktionale Seite des Romans beschrieben. Ungeschützt werden Sentimentalitäten (etwa das »Aquarellbildchen«, $\mathrm{AdB} 3$, 148) und Ressentiments (zum Beispiel gegen Walther Rathenau, AdB 3, 21) offengelegt; Erzähler und Figur überblenden sich:

[D]er Räuberroman [ist] Walsers gescheitestes und gewagtestes Werk, ein Selbstbildnis und eine Selbstuntersuchung von absoluter Unbestechlichkeit, in welcher sowohl der Verfasser der Krankengeschichte als auch deren Subjekt die Stelle des Autors einnehmen. [...] Mit einem sang froid sondergleichen gibt er Rechenschaft über den mutmaßlichen Ursprung seines Leidens in einer fast nur aus lauter kleinen Vernachlässigungen bestehenden Erziehung, darüber, wie er, als fünfzigjähriger Mann, immer noch das Kind und den Knaben in sich spüre, über das Mädchen, das er gerne gewesen wäre, über die Genugtuung, die ihm das Tragen einer Schürze verschafft, über die fetischistischen Neigungen des Löffeliliebkosers, über den Verfolgungswahn [...] und über die aus der sexuellen Verkümmerung, wie er wirklich schreibt, entstehende Gefahr der Vertrottelung. Mit seismographischer Präzision registriert er die geringsten Erschütterungen am Rand seines Bewußtseins, verzeichnet Verwerfungen und Kräuselungen in seinen Gedanken und Emotionen, von denen die psychiatrische Wissenschaft selbst heute kaum etwas sich träumen läßt. Von den therapeutischen Angeboten, die der Gemütsarzt dem Räuber macht, hält der Erzähler nicht viel, 
noch weniger von dem Allheilmittel des Glaubens, den er eine »sehr einfache und billige Seelenzuständlichkeit « nennt. ${ }^{50}$

Johannes Harneit und Michel Roth, die bislang einzigen Komponisten, die sich mit dem »Räuber«-Roman auseinandergesetzt haben, sind nicht in die Falle einer einfachen Dramatisierung und der damit verbundenen Banalisierung getappt. Beide wählten den wahrscheinlich einzig möglichen Weg, nämlich Fragmente des Romans auf der Bühne einfach lesen zu lassen und auf illustrierende Verfahren weitgehend zu verzichten.

\subsection{Johannes Harneits Raubgut-Theater}

Der 1963 in Hamburg geborene Johannes Harneit ist ein musikalisches Multitalent. Er ist als Pianist, Dirigent und Komponist tätig und hat in allen Domänen schon einen bedeutenden Leistungsausweis; vor allem im Bereich der Oper ist er ein international gefragter Dirigent. Als Komponist beschäftigt sich Harneit schwerpunktmäßig mit Literatur. Er meidet ausgetretene Pfade und entdeckt gerne nicht oder kaum vertonte Dichterinnen und Dichter; so jüngst auch Else Lasker-Schüler mit der Oper Ichundich nach ihrem gleichnamigen letzten Theaterstück. ${ }^{51}$ Mit Vorliebe kombiniert Harneit auch Texte unterschiedlicher Autoren; so brachte er zum Beispiel 2001 Adolf Wölfli mit dem Dadaisten und Okkultisten Johannes Baader zusammen. ${ }^{52}$

Mit Robert Walser hat sich Johannes Harneit intensiv beschäftigt. Schon 1991 komponierte er die Robert-Walser-Lieder für Frauen- und Männerstimme und Klavier (vgl. Kap. 10.8), 1999 schrieb er Schnee, eine Kantate für Knabenstimme, Mezzosopran, Bass und kleines Orchester, in der er Walser mit Texten anderer Autoren kombinierte, dann 2003 die Kanons nach Robert Walser für Singstimmen und Klavier. Schließlich entstand 2005 das Musiktheater Räuber. 53

$5^{\circ} \quad$ Sebald: Le promeneur solitaire, S. 156 u. 158.

51 UA Staatsoper Hamburg, o3.11.2019.

$5^{2}$ Johannes Harneit: III. Kantate nach Texten von Johannes Baader und Adolf Wölfli für Sopran, verstimmte Instrumente und zwei Sprechstimmen (Zähler und Nenner). UA: 7. April 2001, Zürich, Schauspielhaus.

53 Schon 2004 gestaltete Johannes Harneit zusammen mit dem Ensemble für Neue Musik Zürich die Theatermusik zur Produktion Geschwister Tanner unter der Regie von Anna Viebrock am Schauspielhaus Zürich. Premiere: 21.02.2004, während der letzten Saison unter der Direktion von Christoph Marthaler am Schauspielhaus Zürich. 
Dafür konnte Harneit den Autor, Dramaturgen und Theaterwissenschaftler Torsten Beyer gewinnen, der in den 199oer-Jahren die Hamburger Theaterszene mit innovativen Experimenten belebt hatte, während der RäuberProduktion aber schon erkrankt war. Beyer erlag Ende 2005 mit 39 Jahren einem Krebsleiden. ${ }^{54}$

Das ohnehin experimentell konzipierte Musiktheater wurde durch äußere Umstände während der Proben ins >Dadaistischeく gesteigert, denn für die Uraufführung in der Opera stabile, der Studiobühne der Hamburger Oper, stand wegen eines bevorstehenden Intendantenwechsels kein Geld mehr zur Verfügung. ${ }^{55}$ Deshalb entschied man sich für eine >Räuber«Variante, in der alles >geraubt< werden durfte - Musik, Kostüme, Bilder, Texte etc. - und vieles bis kurz vor der Uraufführung offenstand. ${ }^{56}$ In einem die Produktion vorbereitenden Artikel schreibt Tom R. Schulz:

\begin{abstract}
Zwei Wochen vor der Uraufführung konnte Johannes Harneit beim besten Willen noch nicht sagen, ob sein Stück »Der Räuber« nach Robert Walser nun eine, drei oder acht Stunden dauern werde. [...] Passend zur masochistisch veranlagten »Räuber«-Figur aus Walsers letztem Roman zieht er sublimen Genuß aus den finanziellen Nöten der Hamburgischen Staatsoper, die ihm den Kompositionsauftrag gab, ohne auch noch Geld für die Produktion erübrigen zu können. Die mit Ulrike Bartusch (Stimme) und Wobine Bosch (Tanz) entstehende Inszenierung arbeitet mit fortschreitend magerer werdenden Textausschnitten aus den 24 mikroskopisch klein beschriebenen Blättern, auf denen Walser den »Räuber« schrieb, mit musikalischen und literarischen Zitaten, die falsche Fährten legen sollen, und mit auf Super-acht gedrehten Filmsequenzen als Zwischenspielen. ${ }^{57}$
\end{abstract}

Durchaus karg erscheint auch die >Partitur< des Sikorski-Verlags: Sie besteht aus zwei Teilen, nämlich aus dem 24 Typoskript-Seiten umfassenden Libretto mit meist kurzen Ausschnitten von Walsers Roman und einem Konvolut von Liedern und Klavierstücken Harneits, die scheinbar mit Walser nichts zu tun haben, mithin eine Art >Raubgut< darstellen. Im Typoskript sind von Hand wenige Notizen für einen improvisierenden Pianisten eingetragen, zum

\footnotetext{
54 Witzeling: Torsten Beyer.

55 Im Sommer 2005 wechselte Louwrens Langevoort als Intendant zur Kölner Philharmonie, Simone Young wurde seine Nachfolgerin an der Hamburger Staatsoper.

56 Man konnte sich dabei auch auf Walsers eigenes Vorgehen beziehen, der im »Räuber«Roman ungeschützt das eigene Leben mit all seinen Sentimentalitäten und Ressentiments schreibend beraubt.

57 Schulz: Tugend aus Noten gemacht.
} 
Beispiel $»($ Cluster sff + Ped. $) \ll^{58}$ oder $»($ bricht Klavierstück ab, legt die nicht gespielten Seiten auf den Boden) «.59 Zudem zeigen Nummern im Typoskript an, wann die Lieder und Klavierstücke gespielt werden müssen. All das scheint während der Proben ins Libretto eingefügt und nach der Aufführung in solch behelfsweisem Zustand dem Musikverlag übergeben worden zu sein. Robert Walsers Vorlage wird einerseits streng ritualisiert, indem nach jedem der 24 Blätter, die sich auf die 24 Mikrogrammseiten beziehen, ein kurzer Film gezeigt wird, andererseits wird Walsers Text in alle Richtungen erweitert. Dieser wird vom Pianisten gesprochen, der mit dem Rücken zum Publikum spielt. Torsten Beyer verlangt, dass auch Walsers Streichungen mitgesprochen werden und so der Schreibprozess, sein Verschreiben und Korrigieren mitläuft: »Alle acht Tage $\{$ bad .. $\}$ nahm er eine Douche, \{die ihn $\}$ unter deren Bespritzung er das Negerlein spielte, indem ihn die Berieselung tänzeln machte. $\{$ Auf $\}$ Von dieser Douche vielleicht noch später. ${ }^{60}$

\subsection{Literarischer Raubzug}

Harneit vertonte keine Ausschnitte aus dem »Räuber«-Roman und auch sonst keine Texte von Walser, sondern ausschließlich Literatur anderer Autoren. Was sich auf den ersten Blick wie ein Raubzug durch die deutsche Literatur ausnimmt, erweist sich bei näherem Hinschauen als vertracktes Netzwerk, in das Walser mehrfach eingebunden ist. Folgende sechs unabhängige Stücke werden vom Pianisten und der Sängerin vorgetragen:

1. Meine Seele für Sopran und Klavier nach Georg Heym

2. Beethoven-Skizzen für Klavier

3. Testament, Melodram für Sprechstimme und Klavier nach Rainer Maria Rilke

4. Klavierstück

5. Du auch für Sopran und Klavier nach Heinrich von Kleists >Gegengedicht $<\mathrm{zu}$ Ein gleiches von Johann Wolfgang Goethe

6. Ich nicht für Sopran und Klavier nach Johann Wolfgang Goethe (Spruch Nr. 157 aus Sprichwörtlich)

Georg Heym schrieb das Gedicht Meine Seele 1911, also während der Berliner Zeit von Robert Walser. ${ }^{61}$ Es ist Gologangi alias Erwin Loewenson gewidmet,

\footnotetext{
58 Harneit: Räuber, S. 5 .

59 Ebd., S. 17.

6o Ebd., S. 8. Die Wörter in gebogenen Klammern sind von Walser gestrichener Text. Harneits Grundlage war die Faksimileausgabe von 1986, vgl. Walser: Der Räuber. Roman, S. 44.

61 Heym: Gesammelte Gedichte, S. 13 .
} 
dem späteren Nachlassverwalter von Georg Heym. Carl Seelig, Walsers späterer Vormund und Begleiter, edierte nach dem Zweiten Weltkrieg Heyms Gedichte und schrieb ein Nachwort, in dem er die damals bekannten Fakten zu Heyms kurzem Leben zusammenfasste. Johannes Harneit vertont das Gedicht in expressionistischem Stil mit weitausgreifendem Gesang und dichtem Klaviersatz. So hätten damals in seiner Berliner Zeit auch Walsers Gedichte von einem fortschrittlichen Komponisten vertont werden können.

Ein Jahr nach Räuber wurde am 12. September 2006 am Beethoven-Fest Bonn die Komposition Beethoven-Skizzen für Kammerorchester uraufgeführt. Johannes Harneit verarbeitet darin das $>$ Keßlersche Skizzenbuch $<{ }^{62}$ das Beethoven ungefähr zwischen 1801 und 1802 führte. Die Nummer 2 von Räuber könnte als Vorstudie zum Bonner Auftrag bezeichnet werden. Sie enthält viel >klassisches« Material, das in so rasendem Tempo vorgetragen werden muss, dass Harneit vorsorglich anmerkt: »es können auch Töne wegbleiben und Rhythmen durcheinandergeraten ${ }^{63}{ }^{63}$ Dieses hastige Sammeln von $>$ Diebesgut leitet über zum längsten Musikteil in Räuber, dem Melodram Das Testament.

Das Testament schrieb Rilke 1922 in Zürich, drei Jahre vor Walsers Niederschrift des »Räuber«-Romans. Und ebenso wie dieser wurde Rilkes Text erst Jahrzehnte nach seinem Tod, 1975, publiziert. Dieses Unikum innerhalb von Rilkes Schaffen geht in der Dissoziation des Schreibens noch über Walsers Roman hinaus. Harneit lässt den Text Wort für Wort sprechen, begleitet von meist gehaltenen und weiten Klavierakkorden. 215 Wörter und Namen werden in 24 >Versen< aneinandergereiht. Nur ganz zum Schluss verdichtet sich die Aneinanderreihung der Wörter zur syntaktischen Aussage »keine Musik reicht / an den Reigen «, ${ }^{64}$ was als Selbstreferenz ans eigene Theaterkonzept zu begreifen ist. Für Michael Lentz ist diese Stelle in Das Testament

ein radikaler Sonderfall innerhalb von Rilkes kompromissloser und darum stets vom Scheitern bedrohter Sprachästhetik. Wörter, ohne den Boden der Syntax, in der Auswahl und ihrer Anordnung zum Teil gesteuert über klangliche Mittel (Alliteration, Assonanz, Reim), müssen für sich sprechen und evozieren doch so etwas wie Abläufe, Vorstellungen, Geschehnisse. Erst am Schluss dieses stotternden »Reigens« baut sich wieder eine syntagmatische Ordnung auf, die allerdings sofort wieder negierend torpediert wird: »Spule / spielt langsam aber keine Musik reicht / an den Reigen $« .65$

62 Beethoven: Keßlersches Skizzenbuch.

63 Harneit: Räuber, Nummer 2, S. 1

64 Rilke: Das große Lesebuch, S. 682f.

65 Lentz: Nachwort, S. 721. 
Die beiden letzten Lieder in Harneits Musiktheater Räuber verweisen auf Walsers Beschäftigung mit Heinrich von Kleist und Johann Wolfgang von Goethe. ${ }^{66}$ Vertont wird ein Gegengedicht zu Goethes berühmtem Ein gleiches [Wanderers Nachtlied]. Kleist hatte es auf einen Zettel notiert.

\section{Goethes Original:}

Über allen Gipfeln

Ist Ruh, In allen Wipfeln

Spürest du Kaum einen Hauch;

Die Vögelein schweigen im Walde.

Warte nur, balde

Ruhest du auch. ${ }^{67}$

Kleists Gegengedicht, auf das Johannes Harneit zurückgeht:

Unter allen Zweigen ist Ruh, In allen Wipfeln hörest du

Keinen Laut.

Die Vöglein schlafen im Walde,

Warte nur, balde

Schläfest du auch. ${ }^{68}$

Roland Reuß hat 2005 einen Aufsatz zu Kleists Goethe-Parodie verfasst, in dem er zeigt, dass sich die Anrede des »du « auf Goethe beziehen lässt und dass man »die Schlußwendung des Gedichts durchaus auch als manifeste Todesdrohung lesen « kann. ${ }^{69}$ Reuß weist anhand einer ausführlichen Interpretation von Goethes Original überzeugend nach, wie Kleist das Transzendierende bei Goethe eliminiert:

Die Transformation der Goetheschen Vorlage [...] hat ihre eigentliche Spitze in der Ersetzung des Wörtchens »schweigen« durch »schlafen« und der Wiederholung dieses Verbums am Ende des Gedichts. [...] Was an Gemeinsamkeit mit der Natur am Ende bleibt, ist der Tod. Mors ultima linea rerum est, das ist - für dich - die triste Wahrheit, die der Redende des Kleistschen Gedichts seinem Gegenüber mit auf den Weg gibt. ${ }^{70}$

66 Vgl. Walsers Lenz (SW 3, 109-114).

67 Goethe: Gedichte, S. 71.

68 Zitiert nach dem Faksimile in Reuß: Ein anderes gleiches, S. 67.

69 Ebd., S. 67 f.

70 Ebd., S. 69 . 
Johannes Harneit vertont die Kleist-Parodie ganz im Sinne von Roland Reuß (vgl. Abb. 40). Im ersten Teil des Liedes wird mit chromatisch verwischter Harmonik noch auf die zahlreichen Vertonungen von Goethes Wanderers Nachtlied angespielt, auch irreale Vogelrufe der im Gegengedicht schlafenden Vögel werden in der obersten Diskantlage des Klaviers imitiert. Zum Schluss stellt Harneit die Todesdrohung plakativ in den Vordergrund - mit einfachen Quint-Oktav-Sprüngen der Sängerin und einer Klavierbegleitung in der tiefsten Klavierlage, wobei noch »2maliges stummes Nachgreifen verlangt« wird, so als müsste der Schlafende in einen definitiven Schlaf >gedrückt $<$ werden. ${ }^{71}$

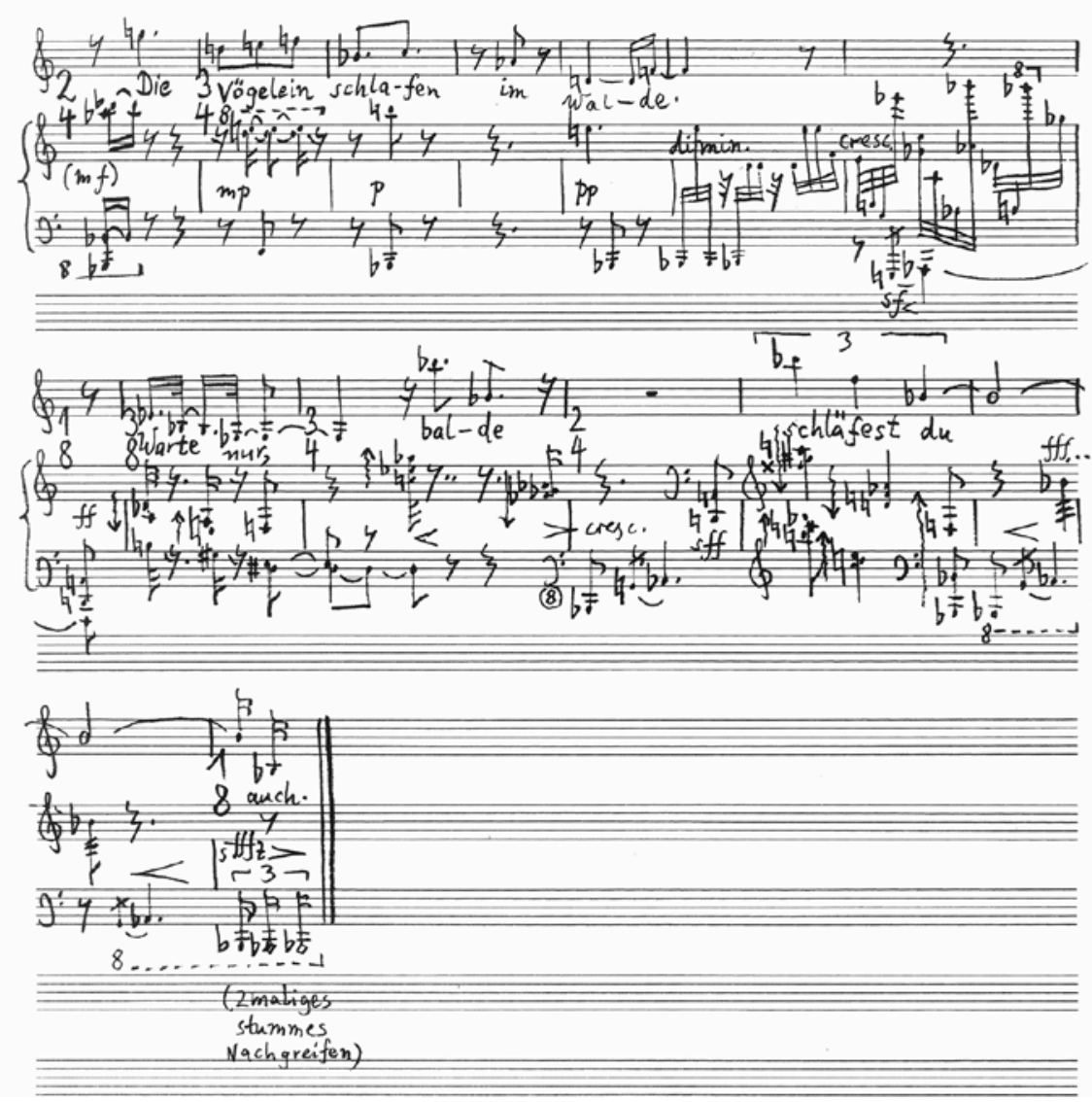

Abb. 40 Johannes Harneit: Über allen Wipfeln, S. 2

71 Interessanterweise hat Walser in einem Gedicht auf den Tod von Rainer Maria Rilke (Rilke, SW 13, 181f.) ebenfalls Versatzstücke von Goethes Ein gleiches ironisch verwendet: »Schön ist nach getaner Pflicht, / Kämpfer um das Gedicht, / solches ungestörtes Ruhn, / entblößt von des Lebens Wanderschuhn.« 
Geradezu kabarettistisch wird dann im sechsten Lied der vor Selbstsicherheit strotzende Spruch Ich nicht von Goethe vertont.

Was ich nicht weiß,

Macht mich nicht heiß.

Und was ich weiß,

Machte mich heiß,

Wenn ich nicht wüßte,

Wie's werden müßte. ${ }^{72}$

Die banalen Reimwörter »weiß« und »heiß« werden von der Sängerin in Überlänge gehalten; das zweite »weiß« muss sie über einer hüpfend bewegten Klavierbewegung sogar während 19 Takten gerade durchsingen! Die artikulierte Selbstsicherheit wird hier mit musikalischer Banalität demontiert: »Wenn ich nicht wüßte « folgt auf einer diatonischen Skala nach oben, »Wie’s werden müßte« auf einer ebenso diatonischen Skala wieder nach unten. Eine Spottvertonung, die sich weit vom »Räuber«-Roman entfernt, aber in ihrem Witz doch von Walsers Geist durchdrungen ist.

Harneits Musiktheater-Experiment ist seit der Uraufführung nicht mehr inszeniert worden. Das dadaistisch-provokative Konzept muss bei der Uraufführung unter der Regie von Hans-Jürg Knapp als Gewaltakt gegen das Publikum wahrgenommen worden sein, wie aus einer Kritik von Elisabeth Richter hervorgeht, die auch schildert, wie man sich dieses Stück vorzustellen hat.

Bei Johannes Harneits neuem Musiktheater »Räuber« [...] wird der Zuschauer zu Abstraktion und Phantasie geradezu gezwungen. In alles Material muss er sich seinen eigenen Sinn hineinarbeiten. [...]

Zwei Frauen - die Bedienung Edith und der Vamp Wanda - rezitieren, sie stehen in der Inszenierung von Hans-Jörg Kapp in den vorderen Stuhlreihen. In der Mitte ein Pianist mit Rücken zum Publikum am Flügel, es ist Walsers Räuber, gespielt vom Komponisten Johannes Harneit. Hinten eine Leinwand, auf der sich verschwommen und zuweilen aufklarend zwei Frauengesichter ausmachen lassen, roter Mund, Arm, Haar und anderes Fleisch ganz nah. ${ }^{73}$

72 Goethe: Gedichte, S. 367.

73 Richter: Viel Platz für Phantasie. 


\subsection{Das komponierte Labyrinth: Michel Roths Auseinandersetzungen mit Robert Walser}

Eine spielerische Auseinandersetzung mit Walsers »Räuber«-Roman sucht auch der Schweizer Michel Roth in seinem sechs Jahre nach Johannes Harneit entstandenen Musiktheater. Michel Roth wurde 1976 in Altdorf geboren, studierte in Basel Komposition und Musiktheorie bei Roland Moser und Detlev MüllerSiemens. Schon früh wurde er an der Luzerner Musikhochschule Dozent für Musiktheorie, Komposition und zeitgenössische Musik. 2011 bekam er eine Professur für Komposition und Musiktheorie an der Hochschule für Musik in Basel. Dort ist er auch als Musikwissenschaftler in der Forschungsabteilung tätig. Die Räuber-Fragmente von 2011 sind Roths erste Beschäftigung mit dem experimentellen Musiktheater. Seither hat er sich auch mit Franz Kafka ${ }^{74}$ und Hermann Burger ${ }^{75}$ musikdramatisch auseinandergesetzt. Die RäuberFragmente sind eine Mischung von Melodram und Kammermusik. Sind bei Harneit mindestens noch die Protagonisten Edith, Wanda und der Räuber als Figuren identifizierbar, auch wenn nie ein dialogisches Moment aufkommt, so eliminiert Roth selbst diese letzten Spuren einer dramatischen Erzählung und überträgt die literarischen Form-Prinzipien des Romans in ein komplexes musikalisch-theatralisches Konzept.

Wie Harneit hat sich auch Michel Roth vor der Arbeit am »Räuber«-Roman bereits intensiv mit Robert Walser auseinandergesetzt, nämlich mit der Novelle Der Spaziergang, die zu Walsers wichtigsten Werken zählt (vgl. RWH, 148). Sie ist 1917 erstmals erschienen, zwei Jahre später publizierte Walser eine überarbeitete Fassung im Band Seeland (vgl. Einleitung).

Für die Übersetzung der literarischen Strategien Walsers in musikalische Formprinzipien, die Michel Roth bei seinen Räuber-Fragmenten anstrebt, bildet dieses kantatenähnliche Werk für zwei Baritone und kleines Orchester, 2009 in München uraufgeführt, eine wichtige Vorstufe. ${ }^{76}$ An zentraler Stelle in Walsers Der Spaziergang thematisiert der Ich-Erzähler Walser sein doppeltes Ich:»Ich war nicht mehr ich selber, war ein anderer und doch gerade darum erst recht wieder ich selbst.«(BA 14, 48) Mit seiner Besetzung für zwei Baritone und Orchester macht Roth dieses >doppelte Ich $<$ zur Grundlage der Komposition

74 Im Bau (nach Franz Kafka). Fünfzehn Klangräume nach einem Fragment von Franz Kafka für Sopran, Oboe, Violoncello und Klavier, 2010-2012. UA 14. September 2012, Lucerne Festival.

75 Die künstliche Mutter (nach Hermann Burger) für Sopran, Mezzosopran, Tenor, Bariton, zwei Schauspielerinnen und Ensemble, 2016. UA 2. September 2016, Lucerne Festival.

76 Beide Walser-Werke von Michel Roth wurden in guten Aufnahmen publiziert. Vgl. Roth: Der Spaziergang (2012) und Räuber-Fragmente (2012). 
und entfaltet einen speziellen Zwiegesang zwischen zwei Stimmen in gleicher Lage: Zwei Ichs fallen sich gegenseitig ins Wort, artikulieren versetzt, synchron und asynchron den gleichen Text, sprechen und singen in unterschiedlichsten Varianten. ${ }^{77}$ Die beiden Baritone repräsentieren auch die beiden Fassungen von Der Spaziergang; so singt einer der Baritone zuweilen - ergänzend, korrigierend, kommentierend - die Textvarianten der Erst- bzw. Zweitfassung hinein. Dabei gibt es keine zugewiesenen Rollen, sondern ein changierendes und perennierendes Doppel-Ich. Diese Ich-Aufspaltung zweier Stimmen in der gleichen Tonlage entspricht in verblüffender Weise »Walsers autofiktional geprägter Schreibweise [...], in der sich der reale Autor und die Ich-Figuren gegenseitig so sehr annähern, dass die Fiktion entsteht, sie könnten einander entsprechen.« (RWH, 151f.) Eine zusätzliche Verfremdung schafft Roth mit zwei Megafonen, welche die Sänger im ersten und letzten Teil benutzen müssen. Das Megafon ist eine von Jahrmärkten und Polizeieinsätzen her geprägte, grobe Klangverstärkung, die Roth zum Abschluss des ersten Teils auf die Worte »hoffentlich hat er das jetzt ein für allemal verstanden« (BA 14, 11) genau in dieser aggressiven Bedeutung einsetzt. An anderen Stellen wird durch das Megafon nur geflüstert oder es wird zu einem eigentlichen >Blasmusik«-Instrument. Die Megafone steigern die theatralische Disposition dieser Kantate, bei der die Sänger nicht einfach nur eine Gesangspartie übernehmen, sondern sich selbst als verdoppeltes und zugleich entzweites Ich spielen.

Michel Roth hat zwar nur etwa sieben Prozent von Walsers Spaziergang vertont, angesichts der umfangreichen Erzählung bedeutet dies aber dennoch viel Text. Er wählte drei zusammenhängende Textteile aus (BA 14, 9-11, 24f., 28 sowie SW 7 [2. Fassung], 83-85, 101f., 105f.), die er nur marginal kürzte: Der erste Teil mit dem Anfang von Walsers Novelle nimmt fast zwei Drittel der Komposition in Anspruch; er endet mit der oben schon erwähnten ironischen Selbstreflexion der Wünsche an den Verfasser und den Worten: »Man ersucht ihn, ernsthaft zu bleiben, und hoffentlich hat er das jetzt ein für allemal verstanden.« (BA 14, 11) Der zweite von Roth gewählte Ausschnitt ist der auffällige Vergleich der Kriegskunst mit der Dichtkunst. Der dritte Ausschnitt ist die Waldszene in der Mitte der Novelle, wo sich der Ich-Erzähler »ein ruhiges kleines Grab zu haben « wünscht. Es befällt ihn »ein unsagbares Weltempfinden« und nachdem er »unhörbare Stimmen« zu vernehmen scheint,

77 Ein Werk, das ebenfalls zwei Baritone in doppelbödiger Bedeutung einsetzt, ist die Oper Don Giovanni von W. A. Mozart. Der Verführer Don Giovanni und sein Diener Leporello, der von den Verführungen lebt und ihre Folgen ausbaden muss, singen bei Mozart in der gleichen Stimmlage. 
folgt der rätselhafte Satz: »Töne aus der Vorwelt kamen, von ich weiß nicht woher, an mein Ohr.« (BA 14, 28)

\subsection{5 >Wort-für-Wort<-Vertonung}

Roth wählt eine Vertonungsart, die allen Verästelungen und Abweichungen Walsers eng folgt; jeder Satzteil, ja jedes Wort wird wörtlich genommen. Ein gutes Beispiel ist der zweite Satz des Textes: »Beifügen könnte ich, daß mir im Treppenhaus eine Frau begegnete, die wie eine Spanierin, Peruanerin oder Kreolin aussah.«(BA 14, 9) Schon dieser Satz ist eine inszenierte Abweichung, die der Ich-Erzähler gleich darauf ironisch kommentiert, indem er sich »auf das strengste verbieten« muss, sich »auch nur zwei Sekunden lang bei dieser Brasilianerin oder was sie sonst sein mochte, aufzuhalten; denn [er] darf weder Raum noch Zeit verschwenden.« (BA 14, 9)

Dieser Abweichung folgt Roth >wörtlich< (vgl. Abb. 41): »Beifügen könnte ich, dass« wird von Bariton 1 »zögernd« in den Ausklang eines stehenden Akkords gesungen, wobei »könnte ich « an der Stimmgrenze des Tenors mit der Falsettstimme zu realisieren ist, während das »dass« nur gesprochen wird. Anschließend fährt Bariton 2 »übermütig« mit der zweiten Version von Der Spaziergang (SW 7, 83) weiter, was zu einer syntaktischen Inkongruenz führt: »Im Treppenhaus« wird mit einem Riesensprung der Tuba ins tiefe Register illustriert und bei »begegnete mir eine Frau« setzt das Posaunen-Solo mit einem anzüglich-ordinär klingenden Metall-Dämpfer ein und begleitet den Rest der Stelle mit Aufmerksamkeit heischenden und übertriebenen Effekten (Trillern, Überblasungen, Portamenti, Rasseln). Dazu setzt die aus Westafrika stammende Talking Drum ein, die auf die exotischen Nationalitäten vorbereitet, die der Ich-Erzähler der Frau im Treppenhaus gleich andichten wird. Michel Roth nimmt in seinem >naiven Wort-für-Wort-Vertonen auch auf diese Nationalitäten Bezug: die »Spanierin« und die »Peruanerin« werden mit Kastagnetten, die »Kreolin« mit Maracas begleitet. Gerade das Klischierte dieser Ausdeutungen will auch das Exotisch-Oberflächliche und Verspielte von Walsers Nationalitäten-Kaskade aufzeigen. Dieses Wort-fürWort-Vertonen führt zu einer dissoziativen musikalischen Form, bei der von Moment zu Moment die Instrumentation, der Gesangsstil, die Text-Verteilung auf die beiden Baritone, ja sogar die Kompositionstechnik wechseln können. Aus dem Reichtum der verschiedenen Kompositionstechniken und den lose gefügten Lokalstrukturen ergeben sich viele Bezüge zu Walsers Text, vor allem auch, weil Roth für die bei Walser oft anzutreffenden $>$ Findlinge $<$ - zum Beispiel in Form von Wortneuschöpfungen - überraschende Lösungen findet. 


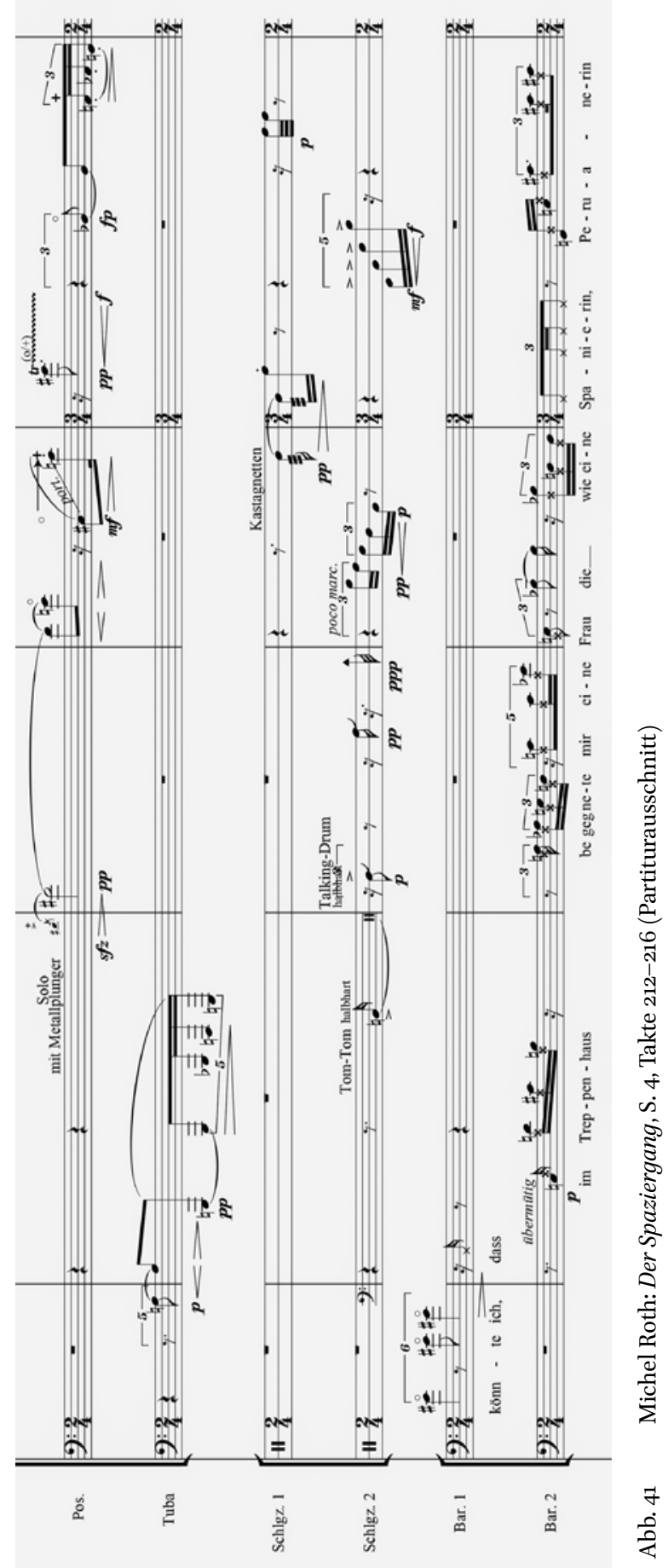




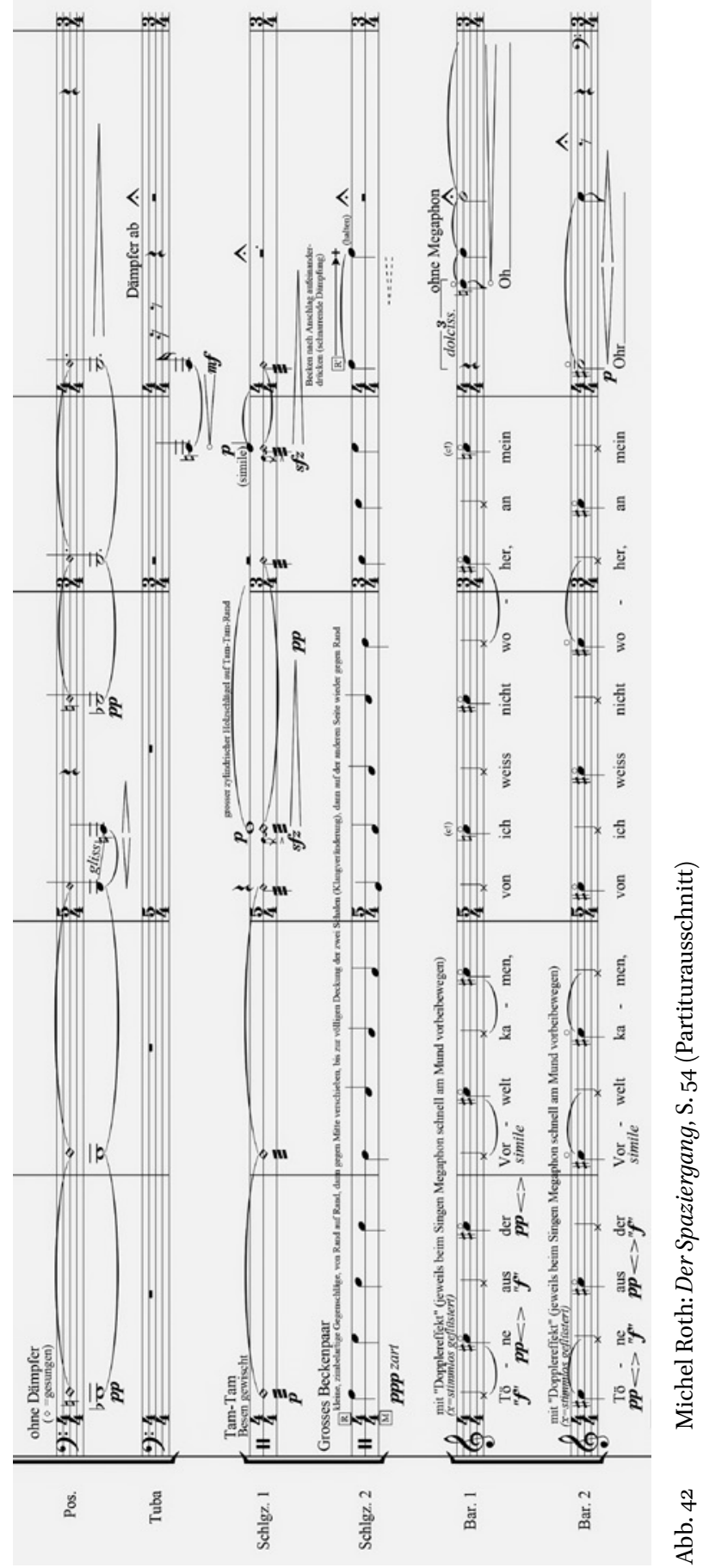


So setzt er den bereits erwähnten Satz mit den »Töne[n] aus der Vorwelt« so um, dass die beiden Baritone jeweils um einen Schlag verschoben zwischen Sprech- und Falsettstimme springen; dabei erklingt in diesem Wechselgesang immer nur das hohe $\mathrm{cis}^{2}$, das durch das rasch vor dem Mund bewegte Megafon überdies verfremdet wird (vgl. Abb. 42). Die Flageoletts in fast allen Orchesterinstrumenten, der in sein Instrument singende Posaunist und die auf- und absteigende Klang->Skala< auf dem großen Beckenpaar erhöhen außerdem die magische Wirkung dieser Stelle - die Musik klingt tatsächlich wie aus einer anderen Welt.

\subsection{Der improvisierende Räuber - Räuber-Fragmente von Michel Roth}

Nach seiner Arbeit zum Spaziergang könnte man vermuten, dass Roth das dort erprobte Prinzip des verdoppelten Sängers für den Ich-Erzähler und den Räuber übernehmen würde. Aber Roth suchte nach einer radikaleren und zugleich abstrakteren Lösung, bei der das Inhaltliche des Romans weitgehend in den Hintergrund tritt.

Zunächst verblüfft die Tatsache, dass es auch bei Roths Auseinandersetzung mit dem »Räuber«-Roman keine eigentliche Partitur gibt, sondern wie bei Harneit nur ein Libretto mit diversen Zusatzteilen und Einzelblättern für die vier Instrumentalisten. Allerdings sind die verschiedenen Teile bei Roth zu einem System verknüpft, in dem alle Beteiligten gleichberechtigt sind und das über oft aleatorische Kommunikationsregeln gesteuert wird. Als Störelement fügt Roth einen >Fremden< ein, den Solisten, der als Improvisierender ein >System im System< darstellt.

Der SOLIST ist der Räuber, wenn auch ohne sichtbare räuberische Attribute. Er muss ein tragbares Instrument spielen, da er während der Aufführung physisch und musikalisch zwischen den verschiedenen Musikern und Musiken umherirrt, sich sein Improvisationsmaterial von ihnen »raubt« (ohne je eigenes einzubringen). Damit kann er zwischen den verschiedenen musikalischen Ebenen vermitteln, aber auch einzelne Protagonisten (musikalisch) überfallen, bedrohen, verzerren oder auch in der Stimme eines anderen »untertauchen ${ }_{.}{ }^{78}$

Mit diesem improvisierenden Solisten, dem vom Komponisten verboten wird, was seine eigentliche künstlerische Identität ausmacht, nämlich Eigenes zu schaffen und zu entwickeln, findet Roth ein hintersinniges Pendant zum Räuber in Walsers Roman, der »Landschaftseindrücke« (AdB 3, 37), »Neigungen«

78 Roth: Räuber-Fragmente, S. 5 . 
(AdB 3, 30) und »Geschichten « (AdB 3, 30) raubt. ${ }^{79}$ Hinzu kommt, dass die Bezeichnung >Solist< täuscht, wird ihm doch kein repräsentatives Podium geboten, sondern wird er »von den übrigen Mitwirkenden mittels $>I n($ ter)vektiven<, konkret Beschimpfungen und musikalischen Drohgebärden, immer wieder vertrieben, läuft also ruhe- und ziellos umher.« ${ }^{80}$

In den ausführlichen Aufführungshinweisen schreibt Michel Roth, dass die Analyse des »Räuber«-Romans von Peter Utz ihm bei derWerkgenese einen entscheidenden Impuls gab. ${ }^{81} \mathrm{Utz}$ bezieht sich bei seiner Untersuchung auf den »labyrinthischen Diskurs « von Manfred Schmeling und die vier Strukturmerkmale, die Schmeling bei der labyrinthischen Erzählung ausmacht: Wiederholung, Widerspruch, Möglichkeit und Reflexion. ${ }^{82}$ Utz erläutert mit diesen Kategorien den labyrinthischen Leseprozess des »Räuber«-Roman, Michel Roth steuert mit ihnen den Kompositions- und den Aufführungsprozess, indem er diese Strukturmerkmale mittels Zitaten den vier Instrumentalisten zuordnet:

- Das Sopransaxophon steht für Widerspruch. Die Partie ist von starken Kontrasten bestimmt, der Saxophonist steuert provokative Kommentare wie $\gg \mathrm{O}$ naive Frage« oder »Das brauche ich Ihnen ja nicht erst noch zu sagen « bei.

- Die Gitarre repräsentiert die Möglichkeit. Sie weist suchende Gesten auf, der Gitarrist äußert leere Versprechen: »Hievon nachher mehr« oder »Wir wollen das im Interesse verhaltener Interessantheit aufsparen«.

- Der Kontrabass ist durch Wiederholung geprägt, repetiert und variiert ähnliche Muster, seine Kommentare sind beschwichtigend oder erläuternd: »Gut, gut, nur weiter« oder »Verzeihen Sie, wenn ich wie die alte Fasnacht erst jetzt daran denke und damit komme.«

- Der Solist steht für die Reflexion. Er nimmt die Ereignisse auf und spinnt sie assoziativ fort. Er trägt nur einen einzigen längeren Kommentar bei.

Roths Komposition ist nicht in einer Partitur, sondern nur in Stimmen notiert: Die Komplexität der vertikalen Überlagerungen ließe sich, wie in der Polyphonie des Spätmittelalters und der Renaissance, in einer Partitur gar nicht darstellen. Roth knüpft nämlich eine ganze Reihe von Verbindungen zwischen den Mitspielenden. Da ist zunächst das mehr oder weniger durchlaufende Libretto des Erzählers, das von sprachlichen Einwürfen der Instrumentalisten

79 Vgl. Gloor: Prekäres Erzählen, S. 146-150 (Unterkapitel »Der raubende Spaziergänger«).

$80 \quad$ Roth: Räuber-Fragmente, S. 5 .

81 Ebd., S. 7. Vgl. Utz: Tanz auf den Rändern, S. 408-423.

82 Schmeling: Der labyrinthische Diskurs. 
regelmäßig unterbrochen wird, wobei oft die Art des Einwurfs offen bleibt und zuweilen auf den Einwurf auch verzichtet werden kann.

In diesen Fluss baut Roth eine Art zweites Uhrwerk ein, dessen Mechanik eigenen Gesetzen folgt: Jedes Mal, wenn im gesprochenen Text das Wort »Räuber« fällt, müssen die Musiker (ausgenommen der Solist) ihr System verlassen und auf einem Zusatzblatt notierte kurze Motive spielen (vgl. Abb. 43). Michel Roth nennt diese Einschübe »A-In(ter)vektiven«. Sie stehen in gänzlich ungewohnten, >irrealen Taktzahlen, die man sonst in Triolen, Quintolen, Septolen etc. notieren würde. Ihre Funktion ist klar: »Diese komplizierte rhythmische Relation soll den metrischen und rhythmischen Fluss der jeweiligen Partie in jedem Fall deutlich unterbrechen. $\aleph^{83}$ Auch hier wird keine exakte Synchronizität zwischen den Instrumentalisten angestrebt; diese ist in einem derartigen System auch gar nicht möglich und kann in der der mobileartigen, auf Interpendenzen beruhenden Form kaum vorgängig geprobt werden.

Die »B-In(ter)vektiven« dienen dazu, den Räuber in Schach zu halten. Sobald der Solist jemandem zu nah kommt, wird er mit einem dieser Schimpfwörter >verjagt<. Zusätzlich sind an einer Stelle »Interpolationen« notiert. Das sind von den Mitspielenden frei verwendbare Einwürfe; Roth schreibt nur die Anzahl der Interpolationen je Abschnitt vor.

Es soll der Eindruck entstehen, als seien alle Verhältnisse zwischen den Mitwirkenden und der Verlauf des Geschehens undurchschaubaren Gesetzen unterworfen oder gar zufällig. Als würden unkontrollierbare Kräfte und Kettenreaktionen den Gang der Erzählung beeinflussen oder geriete der Erzähler selbst immer wieder in den Sog seiner Geschichte oder in Abhängigkeit von seinen Figuren. ${ }^{84}$

Das Labyrinthische wurde bei der Uraufführung im Theater Rigiblick in Zürich am 2. Dezember 2011 erlebbar. Die eigentlich simple Mechanik, mit der auf jedes »Räuber«-Wort mit »In(ter)vektiven« reagiert wird, drängte sich nicht in den Vordergrund, weil diese Einwürfe so fein ausgestaltet sind, dass sie als eine Reihe von Irritationen unter vielen in Erscheinung treten. Ein Grund für das Gelingen des >Labyrinths liegt darin, dass beim Erzähler inhaltliche Bezüge zum »Räuber«-Roman fast zur Gänze weggestrichen sind. Das Libretto von Michel Roth beschränkt sich in der Zitatauswahl auf Verweise und Selbstreflexionen und versucht, die von Peter Utz herausgearbeiteten Parameter Wiederholung, Widerspruch, Möglichkeit und Reflexion zu isolieren. Es sind

83 Roth: Räuber-Fragmente, S. 6.

84 Ebd., S. 8. 
SOPRANSAXOPHON

\section{IN(TER)VEKTIVEN A}
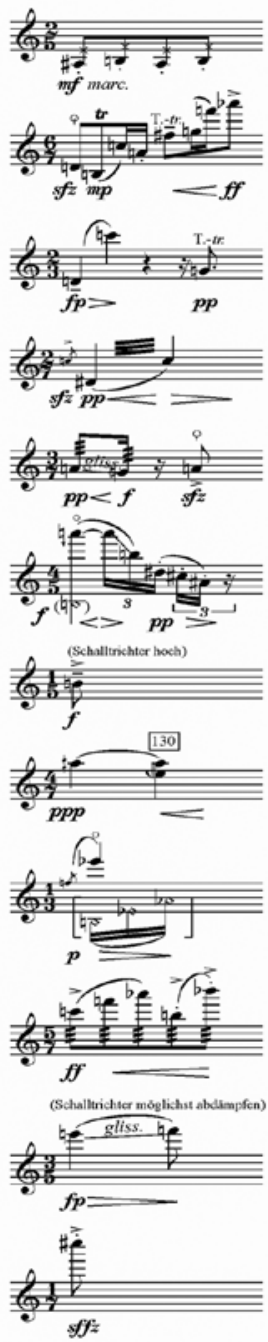

\section{IN(TER)VEKTIVEN B INTERPOLATIONEN}

Petrukio

Löl

Peruaner

Lümmel

Täfeliesser

Löffeliliebkoser

Bürschchen

Fötzel

Tölpel

Knauseri

Grosshans

Grämi

Langweili

O hochgradig intellektueller und zugleich dummer

Idiot

Plagöri

Schuft

Flegeli

Lump

Mädchennachläufer

Trappi

Spitzbube
Einmal zu GIT:

Heraus endlich mit der Sprache! (GIT: Das ist alles, ich schwöre es)

Einmal reagierend auf GIT So geht's, wenn man allerlei verspricht!

Einmal zu KBS:

Nur ruhig

Einmal reagierend auf $K B S$ :

Daraus wird nichts, rundheraus erklärt.

Je einmal zum ERZ:

Das also auch noch.

Übersehen wir das.

Einmal zu ERZ hingehend, ihn unterbrechend:

Weswegen wurde er zum Räuber?

Ohne eine Antwort abzuwarten wieder an Platz zurückkehren

Abb. 43 Michel Roth: Räuber-Fragmente, In(ter)vektiven A, B und Interpolationen, Sopransaxophon 
dies mit Ausnahme der Reflexion substantiell musikalische Parameter, die zumindest der tonalen Grammatik auch in ihre Begrifflichkeit eingeschrieben sind, zum Beispiel >Reprise $<$ (Wiederholung), >Dissonanz (Widerspruch) oder $>$ Trugschluss` (Möglichkeit).

In solcher Reduktion ähnelt das Libretto den Aufführungs- und Spielanweisungen in einer musikalischen Partitur, die das musikalische Geschehen erklären. Statt von einer Vertonung ließe sich hier eher von einer >Vertextung< und Exemplifizierung des musikalischen Labyrinths durch den Schauspieler sprechen. Die Räuber-Fragmente werden auf diese Weise zu einem Spiegelkabinett, das in sich selbst dreht und die Außenwelt kaum mehr einlässt.

\subsection{Das Mikrogramm als musikalische Notation}

Ungefähr in der Mitte von Michel Roths Räuber-Fragmenten gibt es einen musikalisch besonders intensiven Moment, wo alle Beteiligten ihre Rolle tauschen und damit das System aus dem Gleichgewicht bringen. Ausgangspunkt dieser kurzen Szene ist der selbstreflexive Kommentar des Erzählers: »Noch nie, so lange ich am Schreibtisch tätig bin, habe ich so kühn, so unerschrocken begonnen zu schriftstellern. Alle diese Sätze, die ich schon aufs Papier warf, und all diejenigen, die den schon niedergeschriebenen noch folgen. $\ll^{85}$

Daraufhin hört der Solist mit Improvisieren auf und spricht seinen einzigen Text. Es handelt sich dabei um jene für W. G. Sebald zentrale Stelle des »Räuber«-Romans, in der der Erzähler gleichsam als Rezensent seines Buches auftritt: ${ }^{86}$

Ich richte an die Gesunden folgenden Appell: Leset doch nicht immer nur diese gesunden Bücher, machet euch doch auch mit sogenannter krankhafter Literatur näher bekannt, aus der ihr vielleicht wesentliche Erbauung schöpfen könnt. Gesunde Menschen sollten stets gewissermaßen etwas riskieren. Wozu, heilandhagelnochmal, ist man denn gesund? [...] Es ist zum Zähneausreißen, zum Totlachen. (SW 12, 83f.) $)^{87}$

$\mathrm{Zu}$ diesem Text des ansonsten improvisierenden, nun aber musikalisch verstummten Solisten beginnen ihrerseits die drei Instrumentalisten zu

85 Ebd., S. 11.

86 Vgl. Sebald: Le promeneur solitaire, S. 1566 .

87 Die Textfassung von Roth (hier S. 11) unterscheidet sich von der Fassung in SW nur im Verzicht auf das Eszett. 
improvisieren. Michel Roth gibt als Improvisationsvorlage allen denselben Ausschnitt eines Mikrogramms (vgl. Abb. 44). Dabei schränkt Roth den Tonhöhenrahmen der Improvisationen enorm ein, die enge Amplitude von Walsers Mikrogramm-Schrift imitierend. Alle drei Instrumente spielen - trotz ihrer unterschiedlichen Größe - in derselben Lage zwischen $a^{1}$ und $f^{2}$ und verfügen über einen Umfang von einer Quarte bis zu einer Quinte im Fall des Saxophons.

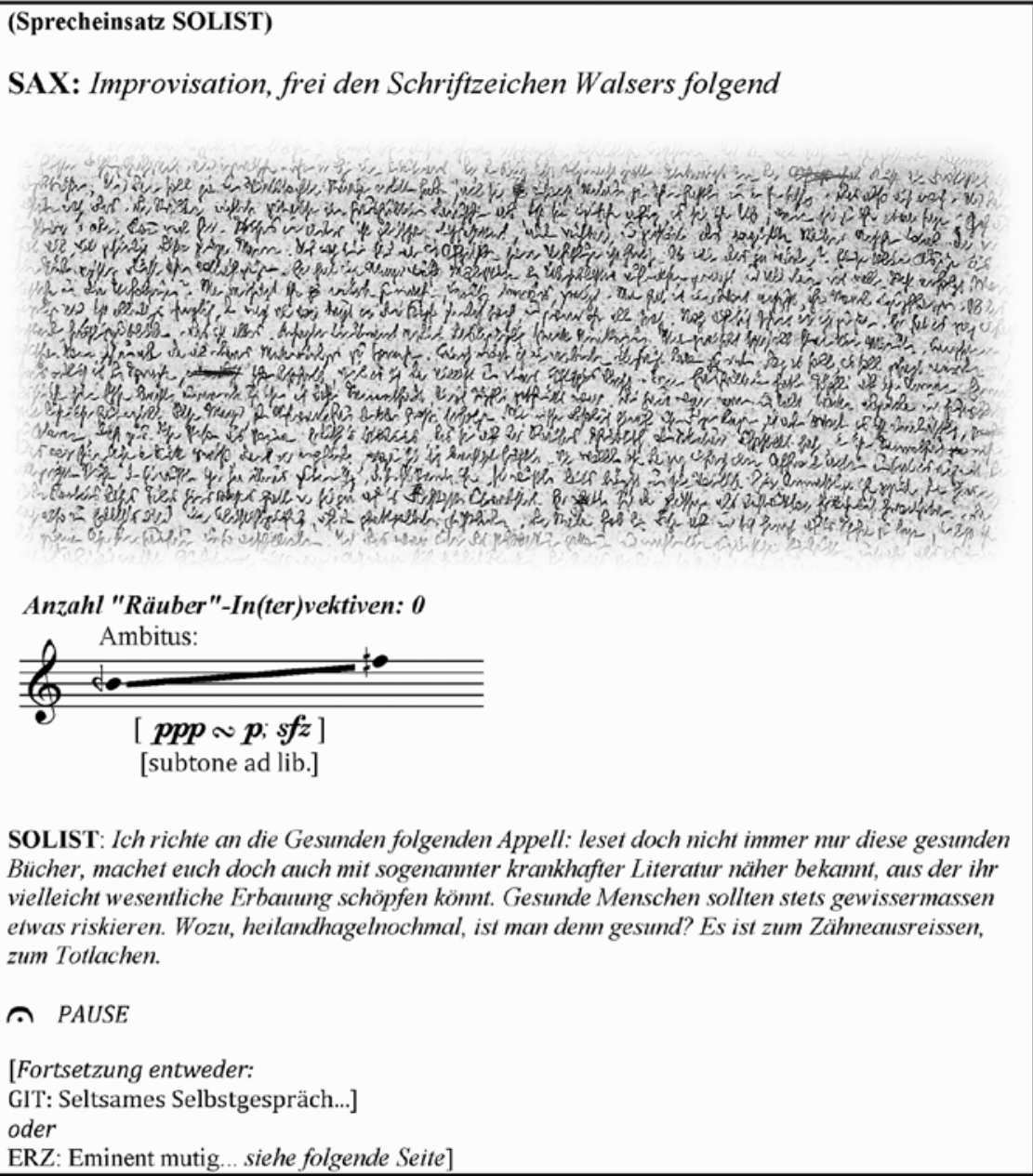

Abb. 44 Michel Roth: Räuber-Fragmente, SAX Improvisation 
Wenn Komponisten auf die Mikrogramm-Schrift mit musikalischer Symbolik reagieren, suchen sie meist nach Auslöschungen oder sich verengenden Tonhöhenstrukturen. Michel Roths Lösung, drei Instrumentalisten das gleiche Mikrogramm als grafische Notation lesen und interpretieren zu lassen, legt mit einer extremen `Engführung \ die enorme Energie der Mikrogramm-Schrift frei.

Dieser Moment höchster Verspannung wird mit einem Einwurf des Gitarristen beendet: »[Seltsames Selbstgespräch, auf welches wir ziemlich gewiss zurückkehren.] « Weil die Interpolation in eckigen Klammern steht, darf der Gitarrist aber auch darauf verzichten. Dann fährt der Erzähler im selbstreflexiven Modus weiter: »Eminent mutig, was ich da sage, nicht wahr? Das Papier verträgt's gut, ob allerdings etwa nachher der Leser oder gar der Durchschnittsleser [- bei Roths radikalen Werk eher: das Publikum oder gar das Durchschnittspublikum - ], ist eine andere Frage. $\ll^{88}$

88 Roth: Räuber-Fragmente, S. 14. 